\title{
CARTA AO EDITOR
}

\section{DADOS EPIDEMIOLÓGICOS DE MALÁRIA NA AMAZÔNIA, POR MUNICÍPIO, REFERENTE A 1992}

Senhor Editor:

Conforme é de seu conhecimento, a Amazônia Legal reúne $98 \%$ dos casos de malária do país, inclusive $99 \%$ dos exames positivos para Plasmodium falciparum são registrados nessa região. Entre os pacientes identificados e tratados fora da Amazônia, as investigações epidemiológicas têm demonstrado que a grande maioria deles (92\%) também contraiu a doença na referida região (casos “importados").

Considerando o exposto, estamos enviando os dados epidemiológicos, por município, de todos os estados que compõem a Amazônia Legal - Acre, Amazonas, Amapá, Maranhão, Mato Grosso, Pará, Rondônia, Roraima e Tocantins, referentes a 1992*, para que Vossa Senhoria considere a possibilidade de publicá-los em nossa Revista. Julgamos que eles poderão ser úteis a pesquisadores, docentes e universitários que se interessam por malária.

Agostinho Cruz Marques

Gerência Técnica de Malária/COAT/DEOPE/FNS

Prédio Anexo MS, $1^{\circ}$ andar, Ala B, sala 116

70058-900 Brasília, DF - Fone: (061) 315- 2178 
Carta ao Editor. Marques AC. Dados epidemiológicos de malária na Amazônia, por município, referente a 1992. Revista da Sociedade Brasileira de Medicina Tropical 26:43-59, jan-mar, 1993.

Tabela I - Acre: dados epidemiológicos da malária por município, em 1992.

\begin{tabular}{|c|c|c|c|c|c|c|c|c|c|c|}
\hline \multirow{2}{*}{ Municípios } & \multirow{2}{*}{$\begin{array}{l}\text { População } \\
\text { IBGE } 1992\end{array}$} & \multicolumn{2}{|c|}{ Amostras de sangue } & \multicolumn{4}{|c|}{ Espécies parasitárias } & \multicolumn{3}{|c|}{ Índices } \\
\hline & & exam & posit & falc & $\%$ falc & vivax & $F+V$ & ILP & IAES & IPA \\
\hline Assis Brasil & 3.077 & 526 & 6 & 3 & 50,0 & 3 & & 1,1 & 17,1 & 1,9 \\
\hline Brasiléia & 20.876 & 2.294 & 222 & 13 & 5,9 & 209 & & 9,7 & 11,0 & 10,6 \\
\hline Cruzeiro do Sul & 68.297 & 9.191 & 2.238 & 1.009 & 45,1 & 1.216 & 13 & 24,3 & 13,5 & 32,8 \\
\hline Feijó & 17.610 & 2.795 & 107 & 9 & 8,4 & 98 & & 3,8 & 15,9 & 6,1 \\
\hline Mâncio Lima & 10.572 & 640 & 48 & 7 & 14,6 & 41 & & 7,5 & 6,1 & 4,5 \\
\hline Manoel Urbano & 5.261 & 769 & 29 & 4 & 13,8 & 25 & & 3,8 & 14,6 & 5,5 \\
\hline Plácido de Castro & 16.254 & 15.236 & 3.052 & 1.215 & 39,8 & 1.833 & 4 & 20,0 & 93,7 & 187,8 \\
\hline Rio Branco & 206.454 & 18.148 & 4.215 & 1.297 & 30,8 & 2.907 & 11 & 23,2 & 8,8 & 20,4 \\
\hline Sena Madureira & 24.222 & 1.998 & 90 & 8 & 8,9 & 82 & & 4,5 & 8,2 & 3,7 \\
\hline Senador Guiomard & 18.849 & 9.844 & 1.232 & 506 & 41,0 & 722 & 4 & 12,5 & 52,2 & 65,4 \\
\hline Tarauacá & 27.615 & 4.909 & 826 & 125 & 15,1 & 698 & 3 & 16,8 & 17,8 & 29,9 \\
\hline Xapuri & 12.186 & 1.605 & 120 & 57 & 47,5 & 63 & & 7,5 & 13,2 & 9,8 \\
\hline Total & 431.273 & 67.955 & 12.185 & 4.253 & 34,9 & 7.897 & 35 & 17,9 & 15,8 & 28,3 \\
\hline
\end{tabular}

Fonte: Boletins da C.R. AC/FNS - População IBGE (estimativa 1992)

exam $=$ examinados; posit $=$ positivos; falc $=P$. falciparum

$\mathrm{F}+\mathrm{V}=$ falciparum + vivax

ILP = índice de lâminas positivas ( $\mathrm{n}^{0}$ de pessoas com exames microscópicos positivos para malária x100 em relação às examinadas); IAES = índice anual de exames de sangue (percentual de pessoas examinadas laboratorialmente em relação à população existente). IPA $=$ incidência parasitária anual ( $n^{\circ}$ de pessoas com exame microscópico positivo por 1000 habitantes).

Tabela 2 - Amapá: dados epidemiológicos de malária por município, em 1992.

\begin{tabular}{|c|c|c|c|c|c|c|c|c|c|c|c|}
\hline \multirow{2}{*}{ Municípios } & \multirow{2}{*}{$\begin{array}{l}\text { População } \\
\text { IBGE } 1992\end{array}$} & \multicolumn{2}{|c|}{ Lâminas } & \multicolumn{5}{|c|}{ Espécies parasitárias } & \multicolumn{3}{|c|}{ Indices } \\
\hline & & exam & posit & falc & $\%$ falc & vivax & $F+V$ & $\mathbf{M}$ & ILP & IAES & IPA \\
\hline Amapá & 8.256 & 1.308 & 177 & 39 & 22,0 & 128 & 10 & & 13,5 & 15,8 & 21,4 \\
\hline Calçoene & 5.477 & 472 & 73 & 22 & 30,1 & 51 & & & 15,5 & 8,6 & 13,3 \\
\hline Ferreira Gomes & 2.369 & 823 & 109 & 46 & 42,2 & 63 & & & 13,2 & 34,7 & 46,0 \\
\hline Laranjal do Jari & 22.175 & 1.431 & 414 & 227 & 54,8 & 183 & 4 & & 28,9 & 6,5 & 18,7 \\
\hline Macapá & 187.638 & 11.026 & 2.963 & 1.063 & 35,9 & 1.892 & 8 & & 26,9 & 5,9 & 15,8 \\
\hline Mazagão & 9.077 & 3.218 & 657 & 269 & 40,9 & 387 & 1 & & 20,4 & 35,5 & 72,4 \\
\hline Oiapoque & 7.826 & 3.004 & 534 & 175 & 32,8 & 358 & 1 & & 17,8 & 38,4 & 68,2 \\
\hline Santana & 55.034 & 6.038 & 1.665 & 458 & 27,5 & 1.200 & 5 & & 27,6 & 11,0 & 30,3 \\
\hline Tartarugalzinho & 4.928 & 2.279 & 377 & 80 & 21,2 & 296 & 1 & & 16,5 & 46,2 & 76,5 \\
\hline Total & 302.780 & 29.599 & 6.969 & 2.379 & 34,1 & 4.558 & 30 & & 23,5 & 9,8 & 23,0 \\
\hline
\end{tabular}

Nenhum município com IPA acima de 100/1000: 0

Municípios com IPA entre 50 e 100/1000: 3

$\begin{array}{ll}\text { Macapá: } & 72,4 \\ \text { Oiapoque: } & 68,2 \\ \text { Tartarugalzinho: } & 76,5\end{array}$

Demais municípios com IPA entre 10 e 49/1000: 6

Amapá: $\quad 21,4$

Calcoene: $\quad 13,3$

Ferreira Gomes: $\quad 46,0$

Laranjal do Jari: $\quad 18,7$

Macapá: $\quad 15,8$

Santana: $\quad 30,3$

$\mathbf{M}=P$. malariae 
Carta ao Editor. Marques AC. Dados epidemiológicos de malária na Amazônia, por município, referente a 1992. Revista da Sociedade Brasileira de Medicina Tropical 26:43-59, jan-mar, 1993.

Tabela 3 - Amazonas: dados epidemiológicos de malária por municipio, em 1992.

\begin{tabular}{|c|c|c|c|c|c|c|c|c|c|c|c|}
\hline \multirow{2}{*}{ Municípios } & \multirow{2}{*}{$\begin{array}{r}\text { População } \\
\text { IBGE } 1992\end{array}$} & \multicolumn{2}{|c|}{ Lâminas } & \multicolumn{5}{|c|}{ Espécies parasitárias } & \multicolumn{3}{|c|}{ Indices } \\
\hline & & exam & posit & falc & $\%$ falc & vivax & $F+V$ & $\mathbf{M}$ & ILP & IAES & IPA \\
\hline Alvarães & 8.971 & 1.887 & 592 & 223 & 37,7 & 368 & 1 & & 31,4 & 21,0 & 66,0 \\
\hline Amaturá & 4.762 & 63 & 5 & & & 5 & & & 7,9 & 1,3 & 1,0 \\
\hline Anamã & 6.102 & & & & & & & & ERR & 0,0 & 0,0 \\
\hline Anori & 8.936 & 214 & 39 & 5 & 12,8 & 34 & & & 18,2 & 2,4 & 4,4 \\
\hline Apuí & 5.456 & 5.349 & 1.287 & 441 & 34,3 & 842 & 4 & & 24,1 & 98,0 & 235,9 \\
\hline Atalaia do Norte & 8.148 & 798 & 257 & 40 & 15,6 & 216 & 1 & & 32,2 & 9,8 & 31,5 \\
\hline Autazes & 16.985 & 1.866 & 641 & 59 & 9,2 & 581 & 1 & & 34,4 & 11,0 & 37,7 \\
\hline Barcelos & 11.356 & 5.384 & 1.121 & 199 & 17,8 & 921 & 1 & & 20,8 & 47,4 & 98,7 \\
\hline Barreirinha & 16.147 & 403 & 8 & 2 & 25,0 & 6 & & & 2,0 & 2,5 & 0,5 \\
\hline Benjamin Constant & 19.098 & 1.317 & 377 & 46 & 12,2 & 329 & 1 & 1 & 28,6 & 6,9 & 19,7 \\
\hline Beruri & 7.508 & 388 & 28 & 3 & 10,7 & 25 & & & 7,2 & 5,2 & 3,7 \\
\hline Boa Vista do Ramos & 7.776 & 170 & 11 & 3 & 27,3 & 8 & & & 6,5 & 2,2 & 1,4 \\
\hline Boca do Acre & 25.227 & 2.830 & 573 & 252 & 44,0 & 319 & 2 & & 20,2 & 11,2 & 22,7 \\
\hline Borba & 16.821 & 2.635 & 605 & 317 & 52,4 & 278 & 10 & & 23,0 & 15,7 & 36,0 \\
\hline Caapiranga & 7.088 & 450 & 65 & 16 & 24,6 & 49 & & & 14,4 & 6,3 & 9,2 \\
\hline Canutama & 11.921 & 4.111 & 849 & 418 & 49,2 & 427 & 4 & & 20,7 & 34,5 & 71,2 \\
\hline Carauari & 19.953 & 2.529 & 592 & 184 & 31,1 & 407 & 1 & & 23,4 & 12,7 & 29,7 \\
\hline Careiro & 36.348 & 6.747 & 2.378 & 425 & 17,9 . & 1.949 & 3 & 1 & 35,2 & 18,6 & 65,4 \\
\hline Careiro Várzea & 18.221 & 616 & 235 & 2 & $0,9^{\circ}$ & 233 & & & 38,1 & 3,4 & 12,9 \\
\hline Coari & 38.442 & 6.895 & 1.283 & 512 & 39,9 & 769 & 1 & 1 & 18,6 & 17,9 & 33,4 \\
\hline Codajás & 13.331 & 128 & 4 & 3 & 75,0 & 1 & & & 3,1 & 1,0 & 0,3 \\
\hline Eirunepê & 20.941 & 349 & 44 & 3 & 6,8 & 41 & & & 12,6 & 1,7 & 2,1 \\
\hline Envira & 16.270 & 358 & 7 & 1 & 14,3 & 6 & & & 2,0 & 2,2 & 0,4 \\
\hline Fonte Boa & 16.761 & 210 & 13 & 3 & 23,1 & 9 & 1 & & 6,2 & 1,3 & 0,8 \\
\hline Guajará & 9.742 & 209 & 32 & & 0,0 & 32 & & & 15,3 & 2,1 & 3,3 \\
\hline Humaitá & 40.430 & 10.319 & 2.713 & 953 & 35,1 & 1.756 & 4 & & 26,3 & 25,5 & 67,1 \\
\hline Ipixuna & 10.275 & 680 & 175 & 121 & 69,1 & 54 & & & 25,7 & 6,6 & 17,0 \\
\hline Iranduba & 19.225 & 1.726 & 559 & 128 & 22,9 & 429 & 1 & 1 & 32,4 & 9,0 & 29,1 \\
\hline Itacoatiara & 55.164 & 1.554 & 237 & 14 & 5,9 & 223 & & & 15,3 & 2,8 & 4,3 \\
\hline Itamarati & 9.315 & 427 & 150 & 78 & 52,0 & 72 & & & 35,1 & 4,6 & 16,1 \\
\hline Itapiranga & 5.211 & 94 & 4 & & 0,0 & 4 & & & 4,3 & 1,8 & 0,8 \\
\hline Japura & 12.406 & 156 & 20 & 1 & 5,0 & 19 & & & 12,8 & 1,3 & 1,6 \\
\hline Juruá & 4.851 & 127 & 4 & 1 & 25,0 & 3 & & & 3,1 & 2,6 & 0,8 \\
\hline Jutaí & 15.485 & 741 & 154 & 59 & 38,3 & 95 & & & 20,8 & 4,8 & 9,9 \\
\hline Labrea & 34.411 & 10.540 & 2.405 & 1.034 & 43,0 & 1.367 & 3 & 1 & 22,8 & 30,6 & 69,9 \\
\hline Manacapuru & 58.310 & 3.073 & 760 & 25 & 3,3 & 735 & & & 24,7 & 5,3 & 13,0 \\
\hline Manaquiri & 8.372 & 659 & 244 & 23 & 9,4 & 221 & & & 37,0 & 7,9 & 29,1 \\
\hline Manaus & 1.056 .741 & 31.563 & 11.718 & 994 & 8,5 & 10.709 & 11 & 4 & 37,1 & 3,0 & 11,1 \\
\hline Manicoré & 38.605 & 3.248 & 712 & 249 & 35,0 & 462 & 1 & & 21,9 & 8,4 & 18,4 \\
\hline Subtotal & 1.741 .112 & 110.813 & 30.901 & 6.837 & 22,1 & 24.004 & 51 & 9 & 27,9 & 6,4 & 17,7 \\
\hline
\end{tabular}


Carta ao Editor. Marques AC. Dados epidemiológicos de malária na Amazônia, por município, referente a 1992. Revista da Sociedade Brasileira de Medicina Tropical 26:43-59, jan-mar, 1993.

Continuação da Tabela 3 - Amazonas: dados epidemiológicos de malária por município, em 1992.

\begin{tabular}{|c|c|c|c|c|c|c|c|c|c|c|c|}
\hline \multirow{2}{*}{ Municípios } & \multirow{2}{*}{$\begin{array}{l}\text { População } \\
\text { IBGE } 1992\end{array}$} & \multicolumn{2}{|c|}{ Lâminas } & \multicolumn{5}{|c|}{ Espécies parasitárias } & \multicolumn{3}{|c|}{ Índices } \\
\hline & & exam & posit & falc & $\%$ falc & vivax & $F+V$ & $\mathbf{M}$ & ILP & IAES & IPA \\
\hline Maraã & 12.043 & 951 & 201 & 4 & 2,0 & 197 & & & 21,1 & 7,9 & 16,7 \\
\hline Maués & 30.522 & 3.780 & 774 & 388 & 50,1 & 385 & 1 & & 20,5 & 12,4 & 25,4 \\
\hline Nhamundá & 13.088 & 169 & 21 & 8 & 38,1 & 13 & & & 12,4 & 1,3 & 1,6 \\
\hline Nova Olinda Norte & 12.951 & 835 & 194 & 89 & 45,9 & 100 & 5 & & 23,2 & 6,4 & 15,0 \\
\hline Nova Airão & 15.328 & 793 & 81 & 12 & 14,8 & 69 & & & 10,2 & 5,2 & 5,3 \\
\hline Novo Aripuanã & 10.971 & 1.112 & 144 & 12 & 8,3 & 130 & 1 & 1 & 12,9 & 10,1 & 13,1 \\
\hline Parintins & 59.099 & 1.198 & 109 & 31 & 28,4 & 78 & & & 9,1 & 2,0 & 1,8 \\
\hline Pauini & 13.437 & 420 & 10 & 6 & 60,0 & 4 & & & 2,4 & 3,1 & 0,7 \\
\hline Presidente Figueiredo & 8.222 & 720 & 155 & 38 & 24,5 & 117 & & & 21,5 & 8,8 & 18,9 \\
\hline Rio Preto da Eva & 7.752 & 649 & 301 & 1 & 0,3 & 296 & 3 & 1 & 46,4 & 8,4 & 38,8 \\
\hline Santa Isabel R. Negro & 12.206 & 1.065 & 207 & 3 & 1,4 & 204 & & & 19,4 & 8,7 & 17,0 \\
\hline Santo Antonio do Içá & 18.318 & 177 & 5 & & 0,0 & 5 & & & 2,8 & 1,0 & 0,3 \\
\hline S. Gabriel da Cachoeira & 23.402 & 3.239 & 1.432 & 16 & 1,1 & 1.414 & 2 & & 44,2 & 13,8 & 61,2 \\
\hline São Paulo de Olivença & 13.946 & 203 & 14 & & 0,0 & 13 & 1 & & 6,9 & 1,5 & 1,0 \\
\hline S. Sebastião do Uatumā & 4.794 & 183 & 2 & & 0,0 & 2 & & & 1,1 & 3,8 & 0,4 \\
\hline Silves & 5.595 & 220 & 3 & & 0,0 & 3 & & & 1,4 & 3,9 & 0,5 \\
\hline Tabatinga & 29.265 & 396 & 59 & 4 & 6,8 & 55 & & & 14,9 & 1,4 & 2,0 \\
\hline Tapauá & 30.187 & 3.876 & 488 & 204 & 41,8 & 283 & 1 & & 12,6 & 12,8 & 16,2 \\
\hline Tefe & 58.668 & 7.821 & 1.340 & 404 & 30,1 & 936 & & & 17,1 & 13,3 & 22,8 \\
\hline Tonantins & 10.295 & & & & & & & & & 0,0 & 0,0 \\
\hline Uarini & 5.414 & 245 & 42 & 17 & 40,5 & 25 & & & 17,1 & 4,5 & 7,8 \\
\hline Urucará & 10.914 & 188 & 8 & 1 & 12,5 & 7 & & & 4,3 & 1,7 & 0,7 \\
\hline Urucurituba & 18.199 & 211 & 10 & 5 & 50,0 & 5 & & & 4,7 & 1,2 & 0,5 \\
\hline Subtotal & 424.616 & 28.451 & 5.600 & 1.243 & 22,2 & 4.341 & 14 & 2 & 19,7 & 6,7 & 13,2 \\
\hline Total & 2.165 .728 & 139.264 & 36.501 & 8.080 & 22,1 & 28.345 & 65 & 11 & 26,2 & 6,4 & 16,9 \\
\hline
\end{tabular}

1 município com IPA acima de 100/1000:

$$
\text { Apui: } 235,9
$$

7 municipios com IPA entre 50 e $99 / 1000$ :

$\begin{array}{ll}\text { Alvarães: } & 66,0 \\ \text { Barcelos: } & 98,7 \\ \text { Canutama: } & 71,2 \\ \text { Careiro: } & 65,2 \\ \text { Amatura: } & 67,1 \\ \text { Labrea: } & 69,9 \\ \text { São Grabriel da Cachoeira: } & 61,2\end{array}$

São Grabriel da Cachoeira: 61,2

25 municípios com IPA entre 10 e 49/1000

16 municípios com IPA entre 1 e $9,9 / 10$ 
Carta ao Editor. Marques AC. Dados epidemiológicos de malária na Amazônia, por município, referente a 1992. Revista da Sociedade Brasileira de Medicina Tropical 26:43-59, jan-mar, 1993.

Tabela 4 - Maranhão: dados epidemiológicos de malária por município, em 1992.

\begin{tabular}{|c|c|c|c|c|c|c|c|c|c|c|c|}
\hline \multirow{2}{*}{ Municípios } & \multirow{2}{*}{$\begin{array}{l}\text { População } \\
\text { IBGE } 1992\end{array}$} & \multicolumn{2}{|c|}{ Lâminas } & \multicolumn{5}{|c|}{ Espécies parasitárias } & \multicolumn{3}{|c|}{ Índices } \\
\hline & & exam & posit & falc & \% falc & vivax & $F+V$ & $\mathbf{M}$ & ILP & IAES & IPA \\
\hline Açailândia & 87.788 & 2.908 & 377 & 158 & 41,9 & 218 & 1 & & 13,0 & 3,3 & 4,3 \\
\hline Afonso Cunha & 4.361 & 368 & 1 & & 0,0 & 1 & & & 0,3 & 8,4 & 0,2 \\
\hline Alcântara & 19.722 & 1.200 & 67 & 4 & 6,0 & 63 & & & 5,6 & 6,1 & 3,4 \\
\hline Aldeias Altas & 19.564 & 1.311 & 14 & 7 & 50,0 & 7 & & & 1,1 & 6,7 & 0,7 \\
\hline Altamira do Maranhão & 19.643 & 567 & 53 & 23 & 43,4 & 27 & 3 & & 9,3 & 2,9 & 2,7 \\
\hline Alto Parnaíba & 10.613 & 573 & 1 & & 0,0 & 1 & & & 0,2 & 5,4 & 0,1 \\
\hline Amarante do Maranhão & 23.710 & 4.824 & 240 & 38 & 15,8 & 199 & 1 & 2 & 5,0 & 20,3 & 10,1 \\
\hline Anajatuba & 19.555 & 454 & 2 & & 0,0 & 2 & & & 0,4 & 2,3 & 0,1 \\
\hline Anapurus & 10.426 & 281 & 16 & 7 & 43,8 & 9 & & & 5,7 & 2,7 & 1,5 \\
\hline Araioses & 44.005 & 1.418 & 2 & 2 & 100,0 & & & & 0,1 & 3,2 & 0,0 \\
\hline Arame & 35.117 & 3.617 & 165 & 53 & 32,1 & 103 & 4 & 5 & 4,6 & 10,3 & 4,7 \\
\hline Arari & 25.200 & 693 & 7 & 2 & 28,6 & 5 & & & 1,0 & 2,8 & 0,3 \\
\hline Axixá & 9.372 & 157 & 8 & & 0,0 & 8 & & & 5,1 & 1,7 & 0,9 \\
\hline Bacabal & 100.576 & 3.499 & 259 & 82 & 31,7 & 169 & 6 & 2 & 7,4 & 3,5 & 2,6 \\
\hline Bacuri & 22.652 & 1.053 & 70 & 3 & 4,3 & 66 & 1 & & 6,6 & 4,6 & 3,1 \\
\hline Balsas & 43.860 & 2.057 & 41 & 24 & 58,5 & 16 & 1 & & 2,0 & 4,7 & 0,9 \\
\hline Barão do Grajaú & 13.593 & 374 & 7 & 7 & 100,0 & & & & 1,9 & 2,8 & 0,5 \\
\hline Barra do Corda & 92.399 & 3.499 & 241 & 85 & 35,3 & 154 & 2 & & 6,9 & 3,8 & 2,6 \\
\hline Barreirinhas & 29.759 & 1.612 & 11 & 6 & 54,5 & 5 & & & 0,7 & 5,4 & 0,4 \\
\hline Benedito Leite & 9.011 & 713 & 2 & 2 & 100,0 & & & & 0,3 & 7,9 & 0,2 \\
\hline Bequimão & 21.646 & 952 & 6 & & 0,0 & 6 & & & 0,6 & 4,4 & 0,3 \\
\hline Bom Jardim & 41.505 & 3.324 & 423 & 151 & 35,7 & 264 & 6 & 2 & 12,7 & 8,0 & 10,2 \\
\hline Brejo & 27.306 & 660 & 11 & & 0,0 & 11 & & & 1,7 & 2,4 & 0,4 \\
\hline Buriti & 22.935 & 1.024 & 26 & 13 & 50,0 & 13 & & & 2,5 & 4,5 & 1,1 \\
\hline Buriti Bravo & 21.485 & 976 & 24 & 13 & 54,2 & 11 & & & 2,5 & 4,5 & 1,1 \\
\hline Cajapió & 11.996 & 344 & 2 & & 0,0 & 2 & & & 0,6 & 2,9 & 0,2 \\
\hline Cajari & 15.977 & 1.169 & 1 & & 0,0 & 1 & & & 0,1 & 7,3 & 0,1 \\
\hline Cândido Mendes & 33.636 & 3.942 & 814 & 89 & 10,9 & 723 & 2 & & 20,6 & 11,7 & 24,2 \\
\hline Cantanhede & 23.943 & 189 & 22 & 7 & 31,8 & 10 & 5 & & 11,6 & 0,8 & 0,9 \\
\hline Carolina & 24.982 & 1.450 & 36 & 17 & 47,2 & 19 & & & 2,5 & 5,8 & 1,4 \\
\hline Carutapera & 31.509 & 4.754 & 464 & 24 & 5,2 & 439 & 1 & & 9,8 & 15,1 & 14,7 \\
\hline Caxias & 147.691 & 6.470 & 229 & 91 & 39,7 & 114 & 17 & 7 & 3,5 & 4,4 & 1,6 \\
\hline Cedral & 15.340 & 292 & & & & & & & 0,0 & 1,9 & 0,0 \\
\hline Chapadinha & 57.254 & 1.882 & 139 & 52 & 37,4 & 83 & 4 & & 7,4 & 3,3 & 2,4 \\
\hline Codó & 111.771 & 3.484 & 222 & 111 & 50,0 & 93 & 8 & 10 & 6,4 & 3,1 & 2,0 \\
\hline Coelho Neto & 41.273 & 1.336 & 16 & 7 & 43,8 & 8 & 1 & & 1,2 & 3,2 & 0,4 \\
\hline Colinas & 35.701 & 1.569 & 27 & 17 & 63,0 & 9 & 1 & & 1,7 & 4,4 & 0,8 \\
\hline Coroatá & 70.665 & 954 & 73 & 22 & 30,1 & 50 & 1 & & 7,7 & 1,4 & 1,0 \\
\hline Cururupu & 41.737 & 1.900 & 177 & 13 & 7,3 & 164 & & & 9,3 & 4,6 & 4,2 \\
\hline Subtotal & 1.439 .278 & 67.849 & 4.296 & 1.130 & 26,3 & 3.073 & 65 & 28 & 6,3 & 4,7 & 3,0 \\
\hline
\end{tabular}


Carta ao Editor. Marques AC. Dados epidemiológicos de malária na Amazônia, por município, referente a 1992. Revista da Sociedade Brasileira de Medicina Tropical 26:43-59, jan-mar, 1993.

Continuação da Tabela 4 - Maranhão: dados epidemiológicos de malária por município, em 1992.

\begin{tabular}{|c|c|c|c|c|c|c|c|c|c|c|c|}
\hline \multirow{2}{*}{ Municipios } & \multirow{2}{*}{$\begin{array}{l}\text { Populaçāo } \\
\text { IBGE } 1992\end{array}$} & \multicolumn{2}{|c|}{ Lâminas } & \multicolumn{5}{|c|}{ Espécies parasitárias } & \multicolumn{3}{|c|}{ Índices } \\
\hline & & exam & posit & falc & $\%$ falc & vivax & $F+V$ & $\mathbf{M}$ & ILP & IAES & IPA \\
\hline Dom Pedro & 19.824 & 272 & 25 & 14 & 56,0 & 10 & & 1 & 9,2 & 1,4 & 1,3 \\
\hline Duque Bacelar & 7.501 & 571 & 4 & & 0,0 & 4 & & 1 & 0,7 & 7,6 & 0,5 \\
\hline Esperantinópolis & 29.016 & 881 & 98 & 27 & 27,6 & 67 & 3 & & 11,1 & 3,0 & 3,4 \\
\hline Estreito & 24.229 & 1.266 & 156 & 41 & 26,3 & 113 & 2 & & 12,3 & 5,2 & 6,4 \\
\hline Fortaleza dos Nogueiras & 12.706 & 687 & 3 & & 0,0 & 2 & 1 & & 0,4 & 5,4 & 0,2 \\
\hline Fortuna & 15.893 & 1.012 & 10 & 6 & 60,0 & 4 & & & 1,0 & 6,4 & 0,6 \\
\hline Godofredo Viana & 29.717 & 4.935 & 1.054 & 51 & 4,8 & 1.001 & 2 & & 21,4 & 16,6 & 35,5 \\
\hline Gonçalves Dias & 16.339 & 1.127 & 122 & 34 & 27,9 & 86 & 2 & & 10,8 & 6,9 & 7,5 \\
\hline Governador Archer & 10.130 & 559 & 14 & 6 & 42,9 & 8 & & & 2,5 & 5,5 & 1,4 \\
\hline Gov. Eugênio Barros & 22.814 & 1.977 & 81 & 34 & 42,0 & 47 & & & 4,1 & 8,7 & 3,6 \\
\hline Graça Aranha & 5.923 & 266 & 10 & 2 & 20,0 & 8 & & & 3,8 & 4,5 & 1,7 \\
\hline Grajaú & 55.084 & 5.058 & 127 & 55 & 43,3 & 70 & 1 & 1 & 2,5 & 9,2 & 2,3 \\
\hline Guimarães & 12.373 & 381 & 1 & & 0,0 & 1 & & & 0,3 & 3,1 & 0,1 \\
\hline Humberto de Campos & 20.204 & 1.076 & & & & & & & 0,0 & 5,3 & 0,0 \\
\hline Icatu & 20.761 & 827 & 37 & & 0,0 & 37 & & & 4,5 & 4,0 & 1,8 \\
\hline Igarapé Grande & 14.714 & 145 & 24 & 11 & 45,8 & 13 & & & 16,6 & 1,0 & 1,6 \\
\hline Imperatriz & 288.973 & 11.301 & 1730 & 723 & 41,8 & 924 & 51 & 32 & 15,3 & 3,9 & 6,0 \\
\hline Itapecuru Mirim & 43.609 & 751 & 56 & 18 & 32,1 & 35 & 3 & & 7,5 & 1,7 & 1,3 \\
\hline João Lisboa & 54.390 & 2.259 & 412 & 95 & 23,1 & 312 & 4 & 1 & 18,2 & 4,2 & 7,6 \\
\hline Joselândia & 15.157 & 954 & 90 & 47 & 52,2 & 43 & & & 9,4 & 6,3 & 5,9 \\
\hline Lago da Pedra & 47.079 & 827 & 200 & 95 & 47,5 & 98 & 7 & & 24,2 & 1,8 & 4,2 \\
\hline Lago do Junco & 19.312 & 318 & 13 & 5 & 38,5 & 7 & 1 & & 4,1 & 1,6 & 0,7 \\
\hline Lago Verde & 13.503 & 934 & 21 & 7 & 33,3 & 12 & & 2 & 2,2 & 6,9 & 1,6 \\
\hline Lima Campos & 12.383 & 81 & 15 & 7 & 46,7 & 8 & & & 18,5 & 0,7 & 1,2 \\
\hline Loreto & 9.592 & 549 & & & & & & & 0,0 & 5,7 & 0,0 \\
\hline Luis Domingues & 9.502 & 1.831 & 230 & 11 & 4,8 & 219 & & & 12,6 & 19,3 & 24,2 \\
\hline Magalhāes de Almeida & 11.526 & 261 & 1 & & 0,0 & 1 & & & 0,4 & 2,3 & 0,1 \\
\hline Mata Roma & 10.687 & 407 & 15 & 3 & 20,0 & 12 & & & 3,7 & 3,8 & 1,4 \\
\hline Matinha & 18.651 & 913 & 31 & 10 & 32,3 & 19 & 2 & & 3,4 & 4,9 & 0,7 \\
\hline Matões & 25.370 & 948 & 11 & 4 & 36,4 & 7 & & & 1,2 & 3.7 & 0,4 \\
\hline Mirador & 17.253 & 1.094 & 28 & 15 & 53,6 & 13 & & & 2,6 & 6,3 & 1,6 \\
\hline Miranda do Norte & 14.807 & 158 & 19 & 2 & 10,5 & 17 & & & 12,0 & 1,1 & 1,3 \\
\hline Mirinzal & 16.943 & 435 & 9 & & 0,0 & 9 & & & 2,1 & 2,6 & 0,5 \\
\hline Monção & 25.730 & 3.586 & 42 & 17 & 40,5 & 25 & & & 1,2 & 13,9 & 1,6 \\
\hline Montes Altos & 19.881 & 916 & 45 & 18 & 40,0 & 26 & 1 & & 4,9 & 4,6 & 2,3 \\
\hline Morros & 18.741 & 1.449 & 16 & 1 & 6,3 & 15 & & & 1,1 & 7,7 & 0,9 \\
\hline Nina Rodrigues & 7.402 & 579 & 6 & 4 & 66,7 & 2 & & & 1,0 & 7,8 & 0,8 \\
\hline Nova Iorque & 4.951 & 275 & 1 & 1 & 100,0 & & & & 0,4 & 5,6 & 0,2 \\
\hline Subtotal & 1.022 .670 & 51.866 & 4.757 & 1.364 & 28,7 & 3.275 & 80 & 38 & 9,2 & 5,1 & 4,7 \\
\hline
\end{tabular}


Carta ao Editor. Marques AC. Dados epidemiológicos de malária na Amazônia, por município, referente a 1992. Revista da Sociedade Brasileira de Medicina Tropical 26:43-59, jan-mar, 1993.

Continuação da Tabela 4 - Máranhão: dados epidemiológicos de malária por município, em 1992.

\begin{tabular}{|c|c|c|c|c|c|c|c|c|c|c|c|}
\hline \multirow{2}{*}{ Municípios } & \multirow{2}{*}{$\begin{array}{l}\text { População } \\
\text { IBGE } 1992\end{array}$} & \multicolumn{2}{|c|}{ Lâminas } & \multicolumn{5}{|c|}{ Espécies parasitárias } & \multicolumn{3}{|c|}{ Índices } \\
\hline & & exam & posit & falc & $\%$ falc & vivax & $F+V$ & $\mathbf{M}$ & ILP & IAES & IPA \\
\hline Olho D'Água das Cunhas & 16.330 & 1.398 & 112 & 49 & 43,8 & 57 & 6 & & 8,0 & 8,6 & 6,9 \\
\hline Paço do Lumiar & 58.966 & 1.897 & 12 & 4 & 33,3 & 8 & & & 0,6 & 3,2 & 0,2 \\
\hline Palmeirândia & 15.714 & 443 & 19 & 4 & 21,1 & 13 & 1 & 1 & 4,3 & 2,8 & 1,2 \\
\hline Paraibano & 15.994 & 394 & 13 & 5 & 38,5 & 8 & & & 3,3 & 2,5 & 0,8 \\
\hline Parnarama & 30.980 & 1.454 & 19 & 9 & 47,4 & 10 & & & 1,3 & 4,7 & 0,6 \\
\hline Passagem Franca & 22.671 & 955 & 17 & 8 & 47,1 & 9 & & & 1,8 & 4,2 & 0,7 \\
\hline Pastos Bons & 14.071 & 729 & 21 & 9 & 42,9 & 11 & 1 & & 2,9 & 5,2 & 1,5 \\
\hline Paulo Ramos & 27.082 & 715 & 95 & 20 & 21,1 & 72 & 3 & & 13,3 & 2,6 & 3,5 \\
\hline Pedreiras & 50.712 & 1.146 & 295 & 117 & 39,7 & 156 & 16 & 6 & 25,7 & 2,3 & 5,8 \\
\hline Penalva & 29.490 & 2.851 & 30 & 12 & 40,0 & 17 & 1 & & 1,1 & 9,7 & 1,0 \\
\hline Peri Mirim & 13.588 & 437 & 2 & 1 & 50,0 & 1 & & & 0,5 & 3,2 & 0,1 \\
\hline Pindaré Mirim & 32.142 & 3.018 & 282 & 128 & 45,4 & 144 & 8 & 2 & 9,3 & 9,4 & 8,8 \\
\hline Pinheiro & 83.816 & 3.790 & 650 & 117 & 18,0 & 522 & 3 & 8 & 17,2 & 4,5 & 7,8 \\
\hline Pio XII & 27.616 & 1.430 & 92 & 41 & 44,6 & 41 & 2 & 8 & 6,4 & 5,2 & 3,3 \\
\hline Pirapemas & 16.067 & 269 & 3 & & 0,0 & 1 & 2 & & 1,1 & 1,7 & 0,2 \\
\hline Poção de Pedras & 23.649 & 203 & 28 & 14 & 50,0 & 13 & 1 & & 13,8 & 0,9 & 1,2 \\
\hline Porto Franco & 33.411 & 916 & 133 & 35 & 26,3 & 96 & 2 & & 14,5 & 2,7 & 4,0 \\
\hline Presidente Dutra & 43.021 & 1.370 & 120 & 58 & 48,3 & 60 & 2 & & 8,8 & 3,2 & 2,8 \\
\hline Presidente Juscelino & 6.495 & 563 & 5 & & 0,0 & 5 & & & 0,9 & 8,7 & 0,8 \\
\hline Presidente Vargas & 7.838 & 200 & 2 & & 0,0 & 2 & & & 1,0 & 2,6 & 0,3 \\
\hline Primeira Cruz & 18.495 & 1.792 & & & & & & & 0,0 & 9,7 & 0,0 \\
\hline Riachão & 28.426 & 1.787 & 21 & 8 & 38,1 & 12 & 1 & & 1,2 & 6,3 & 0,7 \\
\hline Rosário & 42.299 & 639 & 5 & 2 & 40,0 & 3 & & & 0,8 & 1,5 & 0,1 \\
\hline Sambaiba & 5.752 & 549 & 1 & & 0,0 & 1 & & & 0,2 & 9,5 & 0,2 \\
\hline Santa Helena & 25.921 & 4.262 & 671 & 193 & 28,8 & 475 & 3 & & 15,7 & 16,4 & 25,9 \\
\hline Santa Inês & 66.246 & 6.434 & 308 & 126 & 40,9 & 169 & 13 & & 4,8 & 9,7 & 4,6 \\
\hline Santa Luzia & 119.779 & 12.421 & 1.843 & 614 & 33,3 & 1195 & 29 & 5 & 14,8 & 10,4 & 15,4 \\
\hline Santa Luzia do Paruá & 48.585 & 4.136 & 717 & 150 & 20,9 & 563 & 2 & 2 & 17,3 & 8,5 & 14,8 \\
\hline Santa Quitéria do MA & 21.197 & 1.299 & 29 & 15 & 51,7 & 12 & 2 & & 2,2 & 6,1 & 1,4 \\
\hline Santa Rita & 21.696 & 233 & 2 & 1 & 50,0 & 1 & & & 0,9 & 1,1 & 0,1 \\
\hline Santo Antônio dos Lopes & 17.270 & 941 & 31 & 13 & 41,9 & 17 & 1 & & 3,3 & 5,4 & 1,8 \\
\hline São Benedito do R. Preto & 15.237 & 782 & 11 & 2 & 18,2 & 7 & 2 & & 1,4 & 5,1 & 0,7 \\
\hline São Bento & 28.470 & 616 & 91 & 19 & 20,9 & 63 & 4 & 5 & 14,8 & 2,2 & 3,2 \\
\hline São Bernardo & 24.862 & 702 & 7 & & 0,0 & 7 & & & 1,0 & 2,8 & 0,3 \\
\hline São Domingos do MA & 34.919 & 1.267 & 52 & 18 & 34,6 & 34 & & & 4,1 & 3,6 & 1,5 \\
\hline São Félix de Balsas & 6.261 & 598 & & & & & & & 0,0 & 9,6 & 0,0 \\
\hline Subtotal & 1.095 .068 & 62.636 & 5.739 & 1.792 & 31,2 & 3.805 & 105 & 37 & 9,2 & 5,7 & 5,2 \\
\hline
\end{tabular}


Carta ao Editor. Marques AC. Dados epidemiológicos de malária na Amazônia, por município, referente a 1992. Revista da Sociedade Brasileira de Medicina Tropical 26:43-59, jan-mar, 1993.

Continuação da Tabela 4 - Maranhão: dados epidemiológicos de malária por município, em 1992.

\begin{tabular}{|c|c|c|c|c|c|c|c|c|c|c|c|}
\hline \multirow{2}{*}{ Municipios } & \multirow{2}{*}{$\begin{array}{c}\text { População } \\
\text { IBGE } 1992\end{array}$} & \multicolumn{2}{|c|}{ Lâminas } & \multicolumn{5}{|c|}{ Espécies parasitárias } & \multicolumn{3}{|c|}{ Índices } \\
\hline & & exam & posit & falc & $\%$ falc & vivax & $F+V$ & $\mathbf{M}$ & ILP & IAES & IPA \\
\hline São João Batista & 20.227 & 805 & 6 & 1 & 16,7 & 4 & 1 & & 0,7 & 4,0 & 0,3 \\
\hline São Francisco do MA & 12.875 & 557 & 2 & 1 & 50,0 & 1 & & & 0,4 & 4,3 & 0,2 \\
\hline São José de Ribamar & 75.553 & 1.954 & 46 & 3 & 6,5 & 42 & 1 & & 2,4 & 2,6 & 0,6 \\
\hline São João dos Patos & 24.170 & 569 & 35 & 18 & 51,4 & 14 & 2 & 1 & 6,2 & 2,4 & 1,4 \\
\hline São Luís & 723.959 & 4.784 & 354 & 99 & 28,0 & 244 & 4 & 7 & 7,4 & 0,7 & 0,5 \\
\hline São Luís Gonzaga & 25.903 & 1.064 & 12 & 5 & 41,7 & 6 & 1 & & 1,1 & 4,1 & 0,5 \\
\hline São Mateus do MA & 31.739 & 3.252 & 106 & 46 & 43,4 & 59 & 1 & & 3,3 & 10,2 & 3,3 \\
\hline São Rdo. das Mangabeiras & is $\quad 14.323$ & 896 & 10 & 7 & 70,0 & 3 & & & 1,1 & 6,3 & 0,7 \\
\hline São Vicente Ferrer & 18.348 & 496 & 27 & 7 & 25,9 & 19 & 1 & & 5,4 & 2,7 & 1,5 \\
\hline Sítio Novo & 13.939 & 1.872 & 34 & 15 & 44,1 & 17 & 1 & $\mathbf{l}$ & 1,8 & 13,4 & 2,4 \\
\hline Sucupira do Norte & 10.423 & 578 & 7 & 4 & 57,1 & 3 & & & 1,2 & 5,5 & 0,7 \\
\hline Tasso Fragoso & 6.296 & 549 & 5 & 1 & 20,0 & 3 & 1 & & 0,9 & 8,7 & 0,8 \\
\hline Timbiras & 23.185 & 602 & 32 & 6 & 18,8 & 26 & & & 5,3 & 0,0 & 0,0 \\
\hline Timon & 111.035 & 2.449 & 60 & 17 & 28,3 & 42 & 1 & & 2,4 & 10,6 & 2,6 \\
\hline Tuntum & 36.206 & 2.718 & 131 & 55 & 42,0 & 75 & 1 & & 4,8 & 2,4 & 1,2 \\
\hline Tutóia & 43.588 & 1.410 & 4 & 1 & 25,0 & 3 & & & 0,3 & 3,9 & 0,1 \\
\hline Turiaçu & 40.621 & 6.082 & 1.018 & 298 & 29,3 & 714 & 6 & & 16,7 & 14,0 & 23,4 \\
\hline Urbano Santos & 23.745 & 1.141 & 20 & 10 & 50,0 & 10 & & & 1,8 & 2,8 & 0,5 \\
\hline Vargem Grande & 32.842 & 1.260 & 27 & 13 & 48,1 & 14 & & & 2,1 & 5,3 & 1,1 \\
\hline Viana & 41.957 & 1.683 & 31 & 11 & 35,5 & 17 & 3 & & 1,8 & 5,1 & 0,9 \\
\hline Vitória do Mearim & 50.309 & 6.144 & 84 & 38 & 45,2 & 44 & 1 & 1 & 1,4 & 14,6 & 2,0 \\
\hline Vitorino Freire & 31.128 & 2.339 & 46 & 17 & 37,0 & 24 & 5 & & 2,0 & 4,6 & 0,9 \\
\hline Zé Doca & 58.986 & 6.285 & 1.509 & 472 & 31,3 & 1.007 & $* 19$ & 11 & 24,0 & 20,2 & 48,5 \\
\hline Subtotal & 1.471 .357 & 49.489 & 3.606 & 1.145 & 31,8 & 2.391 & 49 & 21 & 7,3 & 3,4 & 2,5 \\
\hline Total & 5.028 .373 & 231.840 & 18.398 & 5.431 & 29,5 & 12.544 & 299 & 124 & 7,9 & 4,6 & 3,7 \\
\hline
\end{tabular}

* $2 \operatorname{casos} \mathbf{F}+\mathbf{M}$ 
Carta ao Editor. Marques AC. Dados epidemiológicos de malária na Amazônia, por município, referente a 1992. Revista da Sociedade Brasileira de Medicina Tropical 26:43-59, jan-mar, 1993.

Tabela 5 - Mato Grosso: dados epidemiológicos de malária por município, em 1992.

\begin{tabular}{|c|c|c|c|c|c|c|c|c|c|c|c|}
\hline \multirow{2}{*}{ Municípios } & \multirow{2}{*}{$\begin{array}{c}\text { População } \\
\text { IBGE } 1992\end{array}$} & \multicolumn{2}{|c|}{ Lâminas } & \multicolumn{5}{|c|}{ Espécies parasitárias } & \multicolumn{3}{|c|}{ Índices } \\
\hline & & exam & posit & falc & $\%$ falc & vivax & $F+V$ & $\mathbf{M}$ & ILP & IAES & IPA \\
\hline Acorizal & 5.411 & 18 & 12 & 5 & 41,7 & 7 & & & 66,7 & 0,3 & 2,2 \\
\hline Água Boa & 18.837 & 749 & 63 & 3 & 4,8 & 60 & & & 8,4 & 4,0 & 3,3 \\
\hline Alta Floresta & 76.871 & 43.651 & 20.088 & 10.021 & 49,9 & 9.952 & 115 & & 46,0 & 56,8 & 261,3 \\
\hline Alto Araguaia & 10.872 & 29 & 5 & 1 & 20,0 & 4 & & & 17,2 & 0,3 & 0,5 \\
\hline Alto Garças & 8.414 & & & & 0,0 & & & & & 0,0 & 0,0 \\
\hline Alto Paraguai & 14.120 & 1 & 1 & & 0,0 & 1 & & & 100,0 & 0,0 & 0,1 \\
\hline Alto Taquari & 3.152 & & & & & & & & & 0,0 & 0,0 \\
\hline Apiacás & 7.599 & 23.358 & 10.611 & 5.629 & 53 & 4.958 & 24 & & 45,4 & 307,4 & $1.396,4$ \\
\hline Araguainha & 1.418 & 1 & & & & & & & 0,0 & 0,1 & 0,0 \\
\hline Araguaiana & 3.344 & 15 & 1 & & 0,0 & 1 & & & 6,7 & 0,4 & 0,3 \\
\hline Araputanga & 12.325 & 13 & & & & & & & 0,0 & 0,1 & 0,0 \\
\hline Arenápolis & 20.275 & 26 & & & & & & & 0,0 & 0,1 & 0,0 \\
\hline Aripuanã & 16.020 & 13.766 & 4.695 & 1.851 & 39,4 & 2.819 & 25 & & 34,1 & 85,9 & 293,1 \\
\hline Barão do Melgaço & 10.040 & 9 & & & & & & & 0,0 & 0,1 & 0,0 \\
\hline Barra do Bugres & 22.925 & 802 & 80 & 25 & 31,3 & 55 & & & 10,0 & 3,5 & 3,5 \\
\hline Barra do Garças & 46.844 & 480 & 131 & 31 & 23,7 & 100 & & & 27,3 & 1,0 & 2,8 \\
\hline Brasnorte & 7.688 & 1.646 & 479 & 183 & 38,2 & 295 & 1 & & 29,1 & 21,4 & 62,8 \\
\hline Cáceres & 80.241 & 1.701 & 396 & 74 & 18,7 & 322 & & & 23,3 & 2,1 & 4,9 \\
\hline Campinápolis & 13.122 & 32 & 2 & & 0,0 & 2 & & & 6,3 & 0,2 & 0,2 \\
\hline Campo Novo do Parecis & 7.351 & 277 & 44 & 18 & 40,9 & 26 & & & 15,9 & 3,8 & 6,0 \\
\hline Campo Verde & 6.478 & & & & & & & & & 0,0 & 0,0 \\
\hline Canarana & 12.678 & 32 & 7 & & 0,0 & 7 & & & 21,9 & 0,3 & 0,6 \\
\hline Castanheira & 9.460 & 2.377 & 1.080 & 570 & 52,8 & 491 & 19 & & 45,4 & 25,1 & 114,2 \\
\hline Chapada dos Guimarães & 13.130 & 137 & 19 & 8 & 42,1 & 11 & & & 13,9 & 1,0 & 1,4 \\
\hline Cláudia & 9.968 & 3 & 1 & & 0,0 & & 1 & & 33,3 & 0,0 & 0,1 \\
\hline Cocalinho & 5.655 & 394 & 24 & 3 & 12,5 & 21 & & & 6,1 & 7,0 & 4,2 \\
\hline Colider & 32.857 & 6.542 & 2.841 & 1.410 & 49,6 & 1.411 & 20 & & 43,4 & 19,9 & 86,5 \\
\hline Comodoro & 10.054 & 2.949 & 915 & 188 & 20,5 & 726 & 1 & & 31,0 & 29,3 & 91,0 \\
\hline Cuiabá & 425.500 & 6.867 & 2.841 & 1.109 & 39,0 & 1.725 & 7 & & 41,4 & 1,6 & 6,7 \\
\hline Denise & 4.810 & 20 & & & & & & & 0,0 & 0,4 & 0,0 \\
\hline Diamantino & 18.070 & 430 & 128 & 21 & 16,4 & 105 & 2 & & 29,8 & 2,4 & 7,1 \\
\hline Dom Aquino & 8.753 & 3 & 1 & 1 & 100,0 & & & & 33,3 & 0,0 & 0,1 \\
\hline Figueirópolis d'Oeste & 5.277 & 77 & 1 & & 0,0 & 1 & & & 1,3 & 1,5 & 0,2 \\
\hline General Carneiro & 4.333 & & & & & & & & & 0,0 & 0,0 \\
\hline Guarantã do Norte & 33.374 & 32.888 & 15.545 & 7.638 & 49,1 & 7.813 & 94 & & 47,3 & 98,5 & 465,8 \\
\hline Guiratinga & 14.818 & 26 & 13 & 4 & 30,8 & 9 & & & 50,0 & 0,2 & 0,9 \\
\hline Indiavaí & 2.005 & 4 & & & & & & & 0,0 & 0,2 & 0,0 \\
\hline Itauba & 7.911 & 234 & 68 & 23 & 33,8 & 45 & & & 29,1 & 3,0 & 8,6 \\
\hline Itiquira & 8.067 & 6 & 2 & 1 & 50,0 & 1 & & & 33,3 & 0,1 & 0,2 \\
\hline Subtotal & 1.020 .067 & 139.563 & 60.094 & 28.817 & 48,0 & 30.968 & 309 & 0 & 43,1 & 13,7 & 58,9 \\
\hline
\end{tabular}


Carta ao Editor. Marques AC. Dados epidemiológicos de malária na Amazônia, por município, referente a 1992. Revista da Sociedade Brasileira de Medicina Tropical 26:43-59, jan-mar, 1993.

Continuação da Tabela 5 - Mato Grosso: dados epidemiológicos de malária por município, em 1992.

\begin{tabular}{|c|c|c|c|c|c|c|c|c|c|c|c|}
\hline \multirow{2}{*}{ Municípios } & \multirow{2}{*}{$\begin{array}{l}\text { Populaçāo } \\
\text { IBGE } 1992\end{array}$} & \multicolumn{2}{|c|}{ Lâminas } & \multicolumn{5}{|c|}{ Espécies parasitárias } & \multicolumn{3}{|c|}{ Índices } \\
\hline & & exam & posit & falc & $\%$ falc & vivax & $F+V$ & $\mathbf{M}$ & ILP & IAES & IPA \\
\hline Jaciara & 22.755 & 3 & 2 & 1 & 50,0 & 1 & & & 66,7 & 0,0 & 0,1 \\
\hline Jangada & 5.298 & 307 & 103 & 26 & 25,2 & 76 & 1 & & 33,6 & 5,8 & 19,4 \\
\hline Jauru & 13.599 & 178 & 40 & 6 & 15,0 & 34 & & & 22,5 & 1,3 & 2,9 \\
\hline Juara & 23.452 & 2.102 & 758 & 2 & 0,3 & 756 & & & 36,1 & 9,0 & 32,3 \\
\hline Juina & 41.943 & 45.281 & 20.462 & 11.516 & 56,3 & 8.872 & 74 & & 45,2 & 108,0 & 487,9 \\
\hline Juruena & 6.602 & 2.690 & 486 & 176 & 36,2 & 310 & & & 18,1 & 40,7 & 73,6 \\
\hline Juscimeira & 10.789 & 15 & 3 & 1 & 33,3 & 2 & & & 20,0 & 0,1 & 0,3 \\
\hline Lucas do R. Verde & 7.367 & & & & & & & & & 0,0 & 0,0 \\
\hline Luciara & 5.783 & 164 & 9 & 2 & 22,2 & 7 & & & 5,5 & 2,8 & 1,6 \\
\hline Marcelândia & 10.082 & 341 & 130 & 32 & 24,6 & 97 & 1 & & 38,1 & 3,4 & 12,9 \\
\hline Matupã & 13.725 & 62.918 & 29.152 & 15.242 & 52,3 & 13.641 & 269 & & 46,3 & 458,4 & $2.124,0$ \\
\hline Mirassol D'Oeste & 26.654 & 295 & 73 & 17 & 23,3 & 56 & & & 24,7 & 1,1 & 2,7 \\
\hline Nobres & 15.548 & 362 & 108 & 53 & 49,1 & 51 & 4 & & 29,8 & 2,3 & 6,9 \\
\hline Nortelândia & 10.002 & 1.382 & 598 & 157 & 26,3 & 439 & 2 & & 43,3 & 13,8 & 59,8 \\
\hline N. S. do Livramento & 10.572 & 45 & & & & & & & 0,0 & 0,4 & 0,0 \\
\hline Nova Brasilândia & 9.456 & 10 & & & & & & & 0,0 & 0,1 & 0,0 \\
\hline Nova Canaã do Norte & 14.690 & 4.980 & 2.067 & 1.001 & 48,4 & 1.038 & 28 & & 41,5 & 33,9 & 140,7 \\
\hline Nova Mutum & 6.362 & 23 & & & & & & & 0,0 & 0,4 & 0,0 \\
\hline Nova Olímpia & 7.455 & & & & & & & & & 0,0 & 0,0 \\
\hline Nova Xavantina & 18.697 & 597 & 197 & 20 & 10,2 & 173 & 4 & & 3,3 & 3,2 & 10,5 \\
\hline Novo Horizonte do Norte & 4.217 & 13 & & & & & & & 0,0 & 0,3 & 0,0 \\
\hline Novo São Joaquim & 7.474 & 11 & & & & & & & 0,0 & 0,1 & 0,0 \\
\hline Paranaita & 13.469 & 17.728 & 8.653 & 4.845 & 56,0 & 3.731 & 77 & & 48,8 & 131,6 & 642,4 \\
\hline Pedra Preta & 11.125 & 1 & & & & & & & 0,0 & 0,0 & 0,0 \\
\hline Paratinga & 19.157 & 209 & 66 & 13 & 19,7 & 53 & & & 31,6 & 1,1 & 3,4 \\
\hline Peixoto de Azevedo & 51.523 & 76.164 & 33.063 & 18.450 & 55,8 & 14.521 & 92 & & 43,4 & 147,8 & 641,7 \\
\hline Poconé & 30.349 & 300 & 78 & 12 & 15,4 & 64 & 2 & & 26,0 & 1,0 & 2,6 \\
\hline Ponte Branca & 3.753 & 7 & & & & & & & 0,0 & 0,2 & 0,0 \\
\hline Pontes e Lacerda & 37.368 & 12.789 & 5.361 & 1.460 & 27,2 & 3.901 & & & 41,9 & 34,2 & 143,5 \\
\hline Porto Alegre do Norte & 11.043 & 3.505 & 1.256 & 542 & 43,2 & 708 & 6 & & 35,8 & 31,7 & 113,7 \\
\hline Porto dos Gaúchos & 8.811 & 18 & & & & & & & 0,0 & 0,2 & 0,0 \\
\hline Poxoréo & 23.948 & 129 & 52 & 18 & 34,6 & 34 & & & 40,3 & 0,5 & 2,2 \\
\hline Primavera do Leste & 13.236 & 18 & 5 & 1 & 20,0 & 4 & & & 27,8 & 0,1 & 0,4 \\
\hline Reserva do Cabacal & 3.012 & 6 & & & & & & & 0,0 & 0,2 & 0,0 \\
\hline Ribeirão Cascalheira & 9.420 & 277 & 65 & 28 & 43,1 & 37 & & & 23,5 & 2,9 & 6,9 \\
\hline Rio Branco & 11.868 & 254 & 1 & & 0,0 & 1 & & & 0,4 & 2,1 & 0,1 \\
\hline Rondonópolis & 130.086 & 906 & 355 & 99 & 27,9 & 252 & 4 & & 39,2 & 0,7 & 2,7 \\
\hline Rosário do Oeste & 20.072 & 296 & 112 & 19 & 17,0 & 84 & 9 & & 37,8 & 1,5 & 5,6 \\
\hline Subtotal & 690.752 & 234.324 & 103.255 & 53.739 & 52,0 & 48.943 & 573 & 0 & 44,1 & 33,9 & 149,5 \\
\hline
\end{tabular}


Carta ao Editor. Marques AC. Dados epidemiológicos de malária na Amazônia, por município, referente a 1992. Revista da Sociedade Brasileira de Medicina Tropical 26:43-59, jan-mar, 1993.

Continuaçāo da Tabela 5 - Mato Grosso: dados epidemiológicos de malária por município, em 1992.

\begin{tabular}{|c|c|c|c|c|c|c|c|c|c|c|c|}
\hline \multirow{2}{*}{ Municípios } & \multirow{2}{*}{$\begin{array}{r}\text { Populaçāo } \\
\text { IBGE } 1992\end{array}$} & \multicolumn{2}{|c|}{ Lâminas } & \multicolumn{5}{|c|}{ Espécies parasitárias } & \multicolumn{3}{|c|}{ Índices } \\
\hline & & exam & posit & falc & $\%$ falc & vivax & $F+V$ & $\mathbf{M}$ & ILP & IAES & IPA \\
\hline Salto do Céu & 7.131 & 184 & 5 & 1 & 20,0 & 4 & & & 2,7 & 2,6 & 0,7 \\
\hline Santa Terezinha & 9.742 & 1.965 & 462 & 243 & 52,6 & 218 & 1 & & 23,5 & 20,2 & 47,4 \\
\hline S. Antônio do Leverger & 15.688 & 213 & 12 & 6 & 50,0 & 6 & & & 5,6 & 1,4 & 0,8 \\
\hline Sāo Félix do Araguaia & 14.755 & 1.157 & 281 & 68 & 24,2 & 213 & & & 24,3 & 7,8 & 19,0 \\
\hline S. José do R. Claro & 17.669 & 1.490 & 292 & 22 & 7,5 & 269 & 1 & & 19,6 & 8,4 & 16,5 \\
\hline S. José dos Quatro Marcos & s $\quad 22.382$ & 331 & 63 & 26 & 41,3 & 37 & & & 19,0 & 1,5 & 2,8 \\
\hline Sinop & 42.520 & 2.641 & 999 & 358 & 35,8 & 631 & 10 & & 37,8 & 6,2 & 23,5 \\
\hline Sorriso & 19.671 & 483 & 161 & 63 & 39,1 & 85 & 13 & & 33,3 & 2,5 & 8,2 \\
\hline Tangará da Serra & 40.720 & 699 & 194 & 83 & 42,8 & 110 & 1 & & 27,8 & 1,7 & 4,8 \\
\hline Tapurah & 8.182 & 513 & 195 & 23 & 11,8 & 171 & 1 & & 38,0 & 6,3 & 23,8 \\
\hline Terra Nova do Norte & 25.352 & 59.767 & 27.778 & 12.768 & 46,0 & 14.879 & 131 & & 46,5 & 235,7 & $1.095,7$ \\
\hline Tesouro & 4.646 & 1 & & & & & & & 0,0 & 0,0 & 0,0 \\
\hline Torixoreu & 8.377 & 4 & & & & & & & 0,0 & 0,0 & 0,0 \\
\hline Várzea Grande & 172.937 & 50 & 4 & 1 & 25,0 & 3 & & & 8,0 & 0,0 & 0,0 \\
\hline Vera & 12.169 & & & & & & & & & 0,0 & 0,0 \\
\hline Vila B. da S. Trindade & 14.900 & 8.637 & 2.706 & 723 & 26,7 & 1.983 & & & 31,3 & 58,0 & 181,6 \\
\hline Vila Rica & 10.581 & 4.741 & 1.680 & 433 & 25,8 & 1.243 & 4 & & 35,4 & 44,8 & 158,8 \\
\hline Subtotal & 447.422 & 82.876 & 34.832 & 14.818 & 42,5 & 19.852 & 162 & & 42,0 & 18,5 & 77,9 \\
\hline Total & 2.158 .241 & 456.763 & 198.181 & 97.374 & 49,1 & 99.763 & 1.044 & & 43,4 & 21,2 & 91,8 \\
\hline
\end{tabular}


Carta ao Editor. Marques AC. Dados epidemiológicos de malária na Amazônia, por município, referente a 1992. Revista da Sociedade Brasileira de Medicina Tropical 26:43-59, jan-mar, 1993.

Tabela 6 - Pará: dados epidemiológicos de malária por município, em 1992.

\begin{tabular}{|c|c|c|c|c|c|c|c|c|c|c|c|}
\hline \multirow{2}{*}{ Municípios } & \multirow{2}{*}{$\begin{array}{c}\text { População } \\
\text { IBGE } 1992\end{array}$} & \multicolumn{2}{|c|}{ Lâminas } & \multicolumn{5}{|c|}{ Espécies parasitárias } & \multicolumn{3}{|c|}{ Índices } \\
\hline & & exam & posit & falc & $\%$ falc & vivax & $F+V$ & $\mathbf{M}$ & IL.P & IAES & IPA \\
\hline Abaetetuba & 105.489 & 404 & 37 & 4 & 10,8 & 33 & & & 9,2 & 0,4 & 0,4 \\
\hline Acará & 37.582 & 1.976 & 4 & 1 & 25,0 & 3 & & & 0,2 & 5,3 & 0,1 \\
\hline Afuá & 19.375 & 1.146 & 250 & 84 & 33,6 & 166 & & & 21,8 & 5,9 & 12,9 \\
\hline Alenquer & 54.334 & 1.145 & 236 & 54 & 22,9 & 179 & 3 & & 20,6 & 2,1 & 4,3 \\
\hline Almerim & 33.303 & 7.110 & 1.748 & 985 & 56,4 & 747 & 16 & & 24,6 & 21,3 & 52,5 \\
\hline Altamira & 154.153 & 19.203 & 5.508 & 1.819 & 33,0 & 3.663 & 22 & 4 & 28,7 & 12,5 & 35,7 \\
\hline Anajas & 14.785 & 1.763 & 307 & 48 & 15,6 & 258 & 1 & & 17,4 & 11,9 & 20,8 \\
\hline Ananindeua & 92.788 & 8.387 & 147 & 13 & 8,8 & 129 & 5 & & 1,8 & 9,0 & 1,6 \\
\hline Augusto Correa & 31.120 & 7.162 & 694 & & 0,0 & 694 & & & 9,7 & 23,0 & 22,3 \\
\hline Aveiro & 10.981 & 159 & 28 & 19 & 67,9 & 8 & 1 & & 17,6 & 1,4 & 2,5 \\
\hline Bagre & 15.175 & 1.007 & 161 & & 0,0 & 161 & & & 16,0 & 6,6 & 10,6 \\
\hline Baião & 20.547 & 2.739 & 689 & 286 & 41,5 & 399 & 4 & & 25,2 & 13,3 & 33,5 \\
\hline Barcarena & 53.620 & 1.282 & 33 & 12 & 36,4 & 21 & & & 2,6 & 2,4 & 0,6 \\
\hline Belém & 1.313 .883 & 32.822 & 657 & 113 & 17,2 & 532 & 11 & 1 & 2,0 & 2,5 & 0,5 \\
\hline Benevides & 83.701 & 5.235 & 12 & 5 & 41,7 & 7 & & & 0,2 & 6,3 & 0,1 \\
\hline Bom Jesus do Tocantins & 16.619 & 1.040 & 311 & 126 & 40,5 & 185 & & & 29,9 & 6,3 & 18,7 \\
\hline Bonito & 8.530 & 202 & 1 & & 0,0 & 1 & & & 0,5 & 2,4 & 0,1 \\
\hline Bragança & 99.455 & 5.848 & 414 & 4 & 1,0 & 410 & & & 7,1 & 5,9 & 4,2 \\
\hline Brejo Grd. do Araguaia & 11.416 & 454 & 100 & 54 & 54,0 & 46 & & & 22,0 & 4,0 & 8,8 \\
\hline Breves & 75.535 & 1.093 & 180 & 86 & 47,8 & 94 & & & 16,5 & 1,4 & 2,4 \\
\hline Bujaru & 14.404 & 1.025 & 1 & 1 & 100,0 & & & & 0,1 & 7,1 & 0,1 \\
\hline Cachoeira do Arari & 13.622 & 244 & 24 & & 0,0 & 24 & & & 9,8 & 1,8 & 1,8 \\
\hline Cametá & 86.247 & 3.764 & 652 & 160 & 24,5 & 487 & 5 & & 17,3 & 4,4 & 7,6 \\
\hline Capanema & 49.333 & 1.171 & 167 & 11 & 6,6 & 156 & & & 14,3 & 2,4 & 3,4 \\
\hline Capitão Poço & 48.165 & 2.116 & 76 & 19 & 25,0 & 57 & & & 3,6 & 4,4 & 1,6 \\
\hline Castanhal & 110.594 & 5.174 & 59 & 12 & 20,3 & 47 & & & 1,1 & 4,7 & 0,5 \\
\hline Chaves & 17.792 & 2.018 & 385 & 168 & 43,6 & 208 & & & 19,1 & 11,3 & 21,6 \\
\hline Colares & 8.675 & 702 & 36 & & 0,0 & 36 & & & 5,1 & 8,1 & 4,1 \\
\hline Conceição do Araguaia & 59.070 & 2.961 & 783 & 369 & 47,1 & 412 & 2 & & 26,4 & 5,0 & 13,3 \\
\hline Concórdia do Pará & 15.566 & 714 & 5 & 2 & 40,0 & 3 & & & 0,7 & 4,6 & 0,3 \\
\hline Curionópolis & 46.441 & 13.373 & 4.462 & 1.666 & 37,3 & 2.777 & 19 & & 33,4 & 28,8 & 96,1 \\
\hline Curralinho & 15.656 & 1 & & & & & & & 0,0 & 0,0 & 0,0 \\
\hline Curuca & 25.918 & 3.040 & 54 & 1 & 1,9 & 53 & & & 1,8 & 11,7 & 2,1 \\
\hline Dom Elizeu & 56.557 & 957 & 186 & 100 & 53,8 & 85 & 1 & & 19,4 & 1,7 & 3,3 \\
\hline Faro & 13.600 & 189 & 9 & & 0,0 & 9 & & & 4,8 & 1,4 & 0,7 \\
\hline Garrafão do Norte & 21.433 & 2.913 & 64 & 32 & 50,0 & 32 & & & 2,2 & 13,6 & 3,0 \\
\hline Gurupa & 19.831 & 150 & 5 & 5 & 100,0 & & & & 3,3 & 0,8 & 0,3 \\
\hline Igarapé-Açu & 28.164 & 914 & 89 & & 0,0 & 89 & & & 9,7 & 3,2 & 3,2 \\
\hline Igarapé Miri & 41.969 & 232 & 44 & 5 & 11,4 & 39 & & & & & \\
\hline Subtotal & 2.945 .428 & 141.835 & 18.618 & 6.264 & 33,6 & 12.250 & 99 & 5 & 13,1 & 4,8 & 6,3 \\
\hline
\end{tabular}


Carta ao Editor. Marques AC. Dados epidemiológicos de malária na Amazônia, por municipio, referente a 1992. Revista da Sociedade Brasileira de Medicina Tropical 26:43-59, jan-mar, 1993.

Tabela 6 - Pará: dados epidemiológicos de malária por município, em 1992.

\begin{tabular}{|c|c|c|c|c|c|c|c|c|c|c|c|}
\hline \multirow{2}{*}{ Municípios } & \multirow{2}{*}{$\begin{array}{l}\text { População } \\
\text { IBGE } 1992\end{array}$} & \multicolumn{2}{|c|}{ Lâminas } & \multicolumn{5}{|c|}{ Espécies parasitárias } & \multicolumn{3}{|c|}{ Índices } \\
\hline & & exam & posit & falc & $\%$ falc & vivax & $F+V$ & $\mathbf{M}$ & ILP & IAES & IPA \\
\hline Inhangapi & 6.533 & 2.124 & & & & & & & 0,0 & 32,5 & 0,0 \\
\hline Irituia & 28.729 & 697 & 4 & 1 & 25,0 & 3 & & & 0,6 & 2,4 & 0,1 \\
\hline Itaituba & 144.710 & 58.661 & 27.291 & 13.874 & 50,8 & 12.900 & 516 & 1 & 46,5 & 40,5 & 188,6 \\
\hline Itupiranga & 95.751 & 11.856 & 4.597 & 2.234 & 48,6 & 2.354 & 9 & & 38,8 & 12,4 & 48,0 \\
\hline Jacundá & 51.986 & 6.113 & 1.531 & 653 & 42,7 & 873 & 5 & & 25,0 & 11,8 & 29,5 \\
\hline Juriti & 23.195 & 897 & 8 & 5 & 62,5 & 3 & & & 0,9 & 3,9 & 0,3 \\
\hline Limoeiro do Ajuru & 16.927 & 1.587 & 444 & 22 & 5,0 & 422 & & & 28,0 & 9,4 & 26,2 \\
\hline Mãe do Rio & 31.174 & 346 & 14 & 5 & 35,7 & 9 & & & 4,0 & 1,1 & 0,4 \\
\hline Magalhães Barata & 7.615 & 814 & 107 & & 0,0 & 107 & & & 13,1 & 10,7 & 14,1 \\
\hline Marabá & 150.736 & 18.335 & 7.813 & 3.770 & 48,3 & 3.952 & 88 & 3 & 42,6 & 12,2 & 51,8 \\
\hline Maracanã & 26.515 & 6.383 & 854 & 3 & 0,4 & 849 & 2 & & 13,4 & 24,1 & 32,2 \\
\hline Marapanim & 20.571 & 3.324 & 285 & & 0,0 & 285 & & & 8,6 & 16,2 & 13,9 \\
\hline Medicilândia & 43.787 & 576 & 145 & 25 & 17,2 & 118 & 2 & & 25,2 & 1,3 & 3,3 \\
\hline Melgaço & 14.965 & 45 & 1 & & 0,0 & 1 & & & 2,2 & 0,3 & 0,1 \\
\hline Mocajuba & 19.827 & 613 & 70 & 15 & 21,4 & 55 & & & 11,4 & 3,1 & 3,5 \\
\hline Moju & 45.408 & 2.076 & 495 & 128 & 25,9 & 367 & & & 23,8 & 4,6 & 10,9 \\
\hline Monte Alegre & 48.855 & 1.287 & 228 & 114 & 50,0 & 113 & 1 & & 17,7 & 2,6 & 4,7 \\
\hline Muaná & 23.014 & 257 & 25 & & 0,0 & 25 & & & 9,7 & 1,1 & 1,1 \\
\hline Nova Timboteua & 9.347 & 399 & 51 & 2 & 3,9 & 49 & & & 12,8 & 4,3 & 5,5 \\
\hline Obidos & 42.999 & 465 & 31 & 11 & 35,5 & 19 & 1 & & 6,7 & 1,1 & 0,7 \\
\hline Oeiras do Pará & 20.246 & 747 & 20 & 5 & 25,0 & 15 & & & 2,7 & 3,7 & 1,0 \\
\hline Oriximiná & 43.608 & 1.338 & 245 & 60 & 24,5 & 179 & 6 & & 18,3 & 3,1 & 5,6 \\
\hline Ouren & 32.808 & 868 & 38 & 9 & 23,7 & 29 & . & & 4,4 & 2,6 & 1,2 \\
\hline Ourilândia do Norte & 49.031 & 19.137 & 5.490 & 2.657 & 48,4 & 2.802 & 31 & & 28,7 & 39,0 & 112,0 \\
\hline Pacajá & 29.424 & 19.071 & 6.744 & 3.325 & 49,3 & 3.400 & 19 & & 35,4 & 64,8 & 229,2 \\
\hline Paragominas & 109.129 & 7.795 & 1.474 & 942 & 63,9 & 523 & 6 & 3 & 18,9 & 7,1 & 13,5 \\
\hline Parauapebas & 73.819 & 4.686 & 1.478 & 776 & 52,5 & 696 & 6 & & 31,5 & 6,3 & 20,0 \\
\hline Peixe-Boi & 5.758 & 499 & 48 & & 0,0 & 48 & & & 9,6 & 8,7 & 8,3 \\
\hline Porta de Pedras & 17.052 & 1.037 & 52 & 1 & 1,9 & 51 & & & 5,0 & 6,1 & 3,0 \\
\hline Portel & 30.258 & 2.942 & 625 & 227 & 36,3 & 392 & 5 & 1 & 21,2 & 9,7 & 20,7 \\
\hline Porto de $\mathrm{Moz}$ & 16.093 & 643 & 19 & 2 & 10,5 & 17 & & & 3,0 & 4,0 & 1,2 \\
\hline Prainha & 28.781 & 5.146 & 69 & 15 & 21,7 & 54 & & & 1,3 & 17,9 & 2,4 \\
\hline Primavera & 17.552 & 1.823 & 103 & & 0,0 & 103 & & & 5,7 & 10,4 & 5,9 \\
\hline Redenção & 63.527 & 15.716 & 7.239 & 3.432 & 47,4 & 3.730 & 77 & & 46,1 & 24,7 & 114,0 \\
\hline Rio Maria & 26.363 & 959 & 239 & 74 & 31,0 & 165 & & & 24,9 & 3,6 & 9,1 \\
\hline Rondon do Pará & 58.698 & 4.611 & 1.697 & 869 & 51,2 & 826 & 2 & & 36,8 & 7,9 & 28,9 \\
\hline Rurópolis & 24.897 & 1.182 & 387 & 117 & 30,2 & 264 & 6 & & 32,7 & 4,7 & 15,5 \\
\hline Salinópolis & 24.651 & 2.016 & 473 & 1 & 0,2 & 472 & & & 23,5 & 8,2 & 19,2 \\
\hline Subtotal & 1.524 .339 & 207.071 & 70.434 & 33.374 & 47,4 & 36.270 & 782 & 8 & 34,0 & 13,6 & 46,2 \\
\hline
\end{tabular}


Carta ao Editor. Marques AC. Dados epidemiológicos de malária na Amazônia, por município, referente a 1992. Revista da Sociedade Brasileira de Medicina Tropical 26:43-59, jan-mar, 1993.

Tabela 6 - Pará: dados epidemiológicos de malária por município, em 1992.

\begin{tabular}{|c|c|c|c|c|c|c|c|c|c|c|c|}
\hline \multirow{2}{*}{ Municípios } & \multirow{2}{*}{$\begin{array}{c}\text { População } \\
\text { IBGE } 1992\end{array}$} & \multicolumn{2}{|c|}{ Lâminaș } & \multicolumn{5}{|c|}{ Espécies parasitárias } & \multicolumn{3}{|c|}{ Índices } \\
\hline & & exam & posit & falc & $\%$ falc & vivax & $F+V$ & $\mathbf{M}$ & ILP & IAES & IPA \\
\hline Salvaterra & 12.200 & 1.295 & 1 & & 0,0 & 1 & & & 0,1 & 10,6 & 0,1 \\
\hline Santa Cruz do Arari & 4.793 & 65 & & & & & & & 0,0 & 1,4 & 0,0 \\
\hline Santa Isabel do Pará & 35.200 & 2.938 & 10 & 1 & 10,0 & 9 & & & 0,3 & 8,3 & 0,3 \\
\hline Santa Maria das Barreiras & 7.692 & 12.977 & 4.869 & 2.562 & 52,6 & 2.250 & 55 & 2 & 37,5 & 168,7 & 633,0 \\
\hline Santa Maria do Pará & 18.620 & 298 & 15 & & 0,0 & 15 & & & 5,0 & 1,6 & 0,8 \\
\hline Santana do Araguaia & 17.928 & 11.233 & 2.658 & 1.289 & 48,5 & 1.356 & 13 & & 23,7 & 62,7 & 148,3 \\
\hline Santarém & 281.086 & 9.537 & 3.297 & 1.427 & 43,3 & 1.812 & 58 & & 34,6 & 3,4 & 11,7 \\
\hline Santarém Novo & 4.440 & 2.288 & 566 & 10 & 1,8 & 556 & & & 24,7 & 51,5 & 127,5 \\
\hline Santo Antônio do Tauá & 18.453 & 1.960 & 6 & 2 & 33,3 & 4 & & & 0,3 & 10,6 & 0,3 \\
\hline Sāo Caetano de Odivelas & 15.810 & 2.653 & 281 & 1 & 0,4 & 280 & & & 10,6 & 16,8 & 17,8 \\
\hline São Domingos do Capim & 36.404 & 2.788 & 56 & 19 & 33,9 & 37 & & & 2,0 & 7,7 & 1,5 \\
\hline São Félix do Xingu & 35.555 & 5.438 & 1.299 & 589 & 45,3 & 704 & 6 & & 23,9 & 15,3 & 36,5 \\
\hline São Francisco do Pará & 11.839 & 349 & 1 & & 0,0 & 1 & & & 0,3 & 2,9 & 0,1 \\
\hline Sāo Geraldo do Araguaia & 45.138 & 4.498 & 1.367 & 542 & 39,6 & 824 & 1 & & 30,4 & 10,0 & 30,3 \\
\hline São João de Pirabas & 17.314 & 1.189 & 474 & 2 & 0,4 & 472 & & & 39,9 & 6,9 & 27,4 \\
\hline São João do Araguaia & 21.591 & 11.268 & 3.974 & 1.756 & 44,2 & 2.185 & 29 & 4 & 35,3 & 52,2 & 184,1 \\
\hline São Miguel do Guamá & 34.214 & 543 & 137 & 75 & 54,7 & 61 & 1 & & 25,2 & 1,6 & 4,0 \\
\hline S. Sebastião da B. Vista & 15.383 & 313 & 23 & 1 & 4,3 & 22 & & & 7,3 & 2,0 & 1,5 \\
\hline Senador José Porfírio & 54.400 & 5.901 & 1.716 & 746 & 43,5 & 968 & 2 & & 29,1 & 10,8 & 31,5 \\
\hline Soure & 17.417 & 1.422 & 2 & & 0,0 & 2 & & & 0,1 & 8,2 & 0,1 \\
\hline Tailândia & 22.737 & 3.476 & 886 & 45 & 5,1 & 840 & 1 & & 25,5 & 15,3 & 39,0 \\
\hline Tomé-Açu & 41.647 & 1.164 & 79 & 32 & 40,5 & 47 & & & 6,8 & 2,8 & 1,9 \\
\hline Tucuma & 80.354 & 3.491 & 281 & 76 & 27,0 & 201 & 4 & & 8,0 & 4,3 & 3,5 \\
\hline Tucuruí & 86.055 & 18.207 & 7.058 & 2.983 & 42,3 & 4.036 & 37 & 2 & 38,8 & 21,2 & 82,0 \\
\hline Uruara & 68.214 & 793 & 228 & 46 & 20,2 & 182 & & & 38,8 & 1,2 & 3,3 \\
\hline Vigia & 40.291 & 2.228 & 285 & 5 & 1,8 & 280 & & & 12,8 & 5,5 & 7,1 \\
\hline Viseu & 64.346 & 8.913 & 1.976 & 14 & 0,7 & 1.962 & & & 22,2 & 13,9 & 30,7 \\
\hline Xinguara & 44.500 & 1.023 & 407 & 146 & 35,9 & 260 & 1 & & $39,, 8$ & 2,3 & 9,1 \\
\hline Subtotal & 1.153 .621 & 118.248 & 31.952 & $12: 369$ & 38,7 & 19.367 & 208 & 8 & 27,0 & 10,3 & 27,7 \\
\hline Total & 5.623 .388 & 467.154 & 121.004 & 52.007 & 43,0 & 67.887 & 1.089 & 21 & 25,9 & 8,3 & 21,5 \\
\hline
\end{tabular}


Carta ao Editor. Marques AC. Dados epidemiológicos de malária na Amazônia, por município, referente a 1992. Revista da Sociedade Brasileira de Medicina Tropical 26:43-59, jan-mar, 1993.

Tabela 7 - Rondônia: dados epidemiológicos de malária por município, em 1992.

\begin{tabular}{|c|c|c|c|c|c|c|c|c|c|c|c|}
\hline \multirow{2}{*}{ Municípios } & \multirow{2}{*}{$\begin{array}{l}\text { População } \\
\text { IBGE } 1992\end{array}$} & \multicolumn{2}{|c|}{ Lâminas } & \multicolumn{5}{|c|}{ Espécies parasitárias } & \multicolumn{3}{|c|}{ Índices } \\
\hline & & exam & posit & falc & $\%$ falc & vivax & $F+V$ & $\mathbf{M}$ & ILP & IAES & IPA \\
\hline Alta Floresta do Oeste & 40.778 & 2.912 & 770 & 282 & 36,6 & 485 & 3 & & 26,4 & 7,1 & 18,9 \\
\hline Alvorada do Oeste & 24.275 & 6.176 & 1.095 & 283 & 25,8 & 805 & 7 & & 17,7 & 25,4 & 45,1 \\
\hline Ariquemes & 93.416 & 126.801 & 28.753 & 10.765 & 37,4 & 17.855 & 133 & & 22,7 & 135,7 & 307,8 \\
\hline Cabixi & 8.885 & 2.365 & 466 & 110 & 23,6 & 355 & 1 & & 19,7 & 26,6 & 52,4 \\
\hline Cacoal & 82.957 & 4.475 & 634 & 192 & 30,3 & 440 & 2 & & 14,2 & 5,4 & 7,6 \\
\hline Cerejeira & 24.894 & 6.447 & 1.557 & 807 & 51,8 & 740 & 10 & & 24,2 & 25,9 & 62,5 \\
\hline Colorado do Oeste & 41.300 & 3.258 & 946 & 366 & 38,7 & 575 & 5 & & 29,0 & 7,9 & 22,9 \\
\hline Costa Marques & 12.362 & 27.598 & 5.500 & 2.010 & 36,5 & 3.466 & 24 & & 19,9 & 223,2 & 444,9 \\
\hline Espigão D'Oeste & 25.119 & 591 & 145 & 46 & 31,7 & 79 & 20 & & 24,5 & 2,4 & 5,8 \\
\hline Guajará-Mirim & 33.952 & 11.490 & 3.017 & 1.193 & 39,5 & 1.806 & 18 & & 26,3 & 33,8 & 88,9 \\
\hline Jaru & 68.547 & 32.072 & 6.890 & 2.060 & 29,9 & 4.802 & 28 & & 21,5 & 46,8 & 100,5 \\
\hline Ji-Parana & 103.016 & 11.796 & 3.362 & 1.141 & 33,9 & 2.133 & $8 \dot{8}$ & & 28,5 & 11,5 & 32,6 \\
\hline Machadinho D'Oeste & 17.205 & 41.657 & 7.637 & 1.790 & 23,4 & 5.828 & 19 & & 18,3 & 242,1 & 443,9 \\
\hline Nova Brasilândia & 16.664 & 2.025 & 153 & 48 & 31,4 & 105 & & & 7,6 & 12,2 & 9,2 \\
\hline Ouro Preto do Oeste & 90.299 & 18.312 & 3.606 & 1.193 & 33,1 & 2.406 & 7 & & 19,7 & 20,3 & 39,9 \\
\hline Pimenta Bueno & 52.939 & 4.556 & 909 & 262 & 28,8 & 646 & 1 & & 20,0 & 8,6 & 17,2 \\
\hline Porto Velho & 306.906 & 162.658 & 60.203 & 24.283 & 40,3 & 35.577 & 343 & & 37,0 & 53,0 & 196,2 \\
\hline Presidente Medici & 34.125 & 1.845 & 362 & 75 & 20,7 & 287 & & & 19,6 & 5,4 & 10,6 \\
\hline Rolim de Moura & 67.049 & 4.516 & 944 & 284 & 30,1 & 650 & 10 & & 20,9 & 6,7 & 14,1 \\
\hline Santa Luzia do Oeste & 13.123 & 979 & 139 & 39 & 28,1 & 98 & 2 & & 14,2 & 7,5 & 10,6 \\
\hline São Miguel do Guaporé & 24.228 & 15.872 & 3.228 & 890 & 27,6 & 2.328 & 10 & & 20,3 & 65,5 & 133,2 \\
\hline Vila Nova do Mamoré & 7.074 & 15.344 & 5.199 & 2.517 & 48,4 & 2.661 & 21 & & 33,9 & 216,9 & 734,9 \\
\hline Vilhena & 41.305 & 7.191 & 1.911 & 732 & 38,3 & 1.156 & 23 & & 26,6 & 17,4 & 46,3 \\
\hline 10 tai & 1.230 .418 & 510.936 & 137.426 & 51.368 & 37,4 & 85.283 & 775 & & 26,9 & 41,5 & 111,7 \\
\hline
\end{tabular}

Municípios com IPA acima de 100/1000: 7

Municípios com IPA entre 50 e 49/1000: 3
Municípios com IPA entre 10 e 44/1000: 10

Municípios com IPA inferior a 10/1000: 3

Tabela 8 - Roraima: dados epidemiológicos de malária por município, em 1992.

\begin{tabular}{|c|c|c|c|c|c|c|c|c|c|c|c|}
\hline \multirow{2}{*}{ Municípios } & \multirow{2}{*}{$\begin{array}{l}\text { População } \\
\text { IBGE } 1992\end{array}$} & \multicolumn{2}{|c|}{ Lâminas } & \multicolumn{5}{|c|}{ Espécies parasitárias } & \multicolumn{3}{|c|}{ Índices } \\
\hline & & exam & posit & falc & $\%$ falc & vivax & $F+V$ & $\mathbf{M}$ & ILP & IAES & IPA \\
\hline Alto Alegre & 12.541 & 14.772 & 4.986 & 2.110 & 42,3 & 2.777 & 99 & & 33,8 & 118,6 & 400,4 \\
\hline Boa Vista & 156.637 & 29.775 & 10.295 & 4.373 & 42,5 & 5.800 & 122 & & 34,6 & 19,0 & 65,7 \\
\hline Bonfim & 10.107 & 7.950 & 1.955 & 530 & 27,1 & 1.420 & 5 & & 24,6 & 78,7 & 193,4 \\
\hline Caracaraí & 9.392 & 7.238 & 1.299 & 457 & 35,2 & 801 & 41 & & 17,9 & 77,1 & 138,3 \\
\hline Mucajaí & 14.965 & 17.354 & 3.376 & 1.243 & 36,8 & 2.095 & 38 & & 19,5 & 116,0 & 225,6 \\
\hline Normandia & 11.552 & 2.359 & 444 & 183 & 41,2 & 255 & 6 & & 18,8 & 20,4 & 38,4 \\
\hline São João da Baliza & 11.862 & 3.772 & 657 & 125 & 19,0 & 532 & & & 17,4 & 31,8 & 55,4 \\
\hline São Luís do Anauá & 10.396 & 5.659 & 838 & 202 & 24,1 & 636 & & & 14,8 & 54,4 & 80,6 \\
\hline Total & 237.362 & 88.879 & 23.850 & 9.223 & 38,7 & 14.316 & 311 & 0 & 26,8 & 37,4 & 100,5 \\
\hline
\end{tabular}

Municípios com IPA de 100/1000:

$\begin{array}{ll}\text { Alto Alegre: } & 400,4 \\ \text { Mucajaí: } & 225,6 \\ \text { Bonfim: } & 193,4 \\ \text { Caracarai: } & 138,3\end{array}$

Municípios com IPA entre 50 e 99/1000:

$\begin{array}{ll}\text { São Luis do Anauá: } & 80,6 \\ \text { Boa Vista: } & 65,7 \\ \text { São João da Balisa: } & 55,4\end{array}$

Município com IPA abaixo de 50/1000:

Normandia: $\quad 38,4$ 
Carta ao Editor. Marques AC. Dados epidemiológicos de malária na Amazônia, por município, referente a 1992. Revista da Sociedade Brasileira de Medicina Tropical 26:43-59, jan-mar, 1993.

Tabela 9 - Tocantins: dados epidemiológicos de malária por município, em 1992.

\begin{tabular}{|c|c|c|c|c|c|c|c|c|c|c|c|}
\hline \multirow{2}{*}{ Municípios } & \multirow{2}{*}{$\begin{array}{l}\text { População } \\
\text { IBGE } 1992\end{array}$} & \multicolumn{2}{|c|}{ Lâminas } & \multicolumn{5}{|c|}{ Espécies parasitárias } & \multicolumn{3}{|c|}{ Indices } \\
\hline & & exam & posit & falc & \% falc & vivax & $F+V$ & $\mathbf{M}$ & ILP & IAES & IPA \\
\hline Aliança do Tocantins & 6.822 & 347 & & & & & & & 0,0 & 5,1 & 0,0 \\
\hline Almas & 7.831 & 1.207 & 24 & 18 & 75,0 & 6 & & & 2,0 & 15,4 & 3,1 \\
\hline Alvorada & 10.122 & 240 & 4 & & 0,0 & 4 & & & 1,7 & 2,4 & 0,4 \\
\hline Ananás & 11.974 & 2.978 & 199 & 55 & 27,6 & 143 & 1 & & 6,7 & 24,9 & 16,6 \\
\hline Aparecida do R. Negro & 3.301 & 194 & & & & & & & 0,0 & 5,9 & 0,0 \\
\hline Araguacema & 6.438 & 298 & 7 & 6 & 85,7 & 1 & & & 2,3 & 4,6 & 1,1 \\
\hline Araguaçu & 12.610 & 995 & 15 & 5 & 33,3 & 10 & & & 1,5 & 7,9 & 1,2 \\
\hline Araguaína & 106.911 & 2.658 & 554 & 211 & 38,1 & 340 & 3 & & 20,8 & 2,5 & 5,2 \\
\hline Araguatins & 29.136 & 10.102 & 747 & 391 & 52,3 & 355 & 1 & & 7,4 & 34,7 & 25,6 \\
\hline Arapoema & 13.806 & 741 & 21 & 6 & 28,6 & 15 & & & 2,8 & 5,4 & 1,5 \\
\hline Arraias & 12.962 & 869 & & & & & & & 0,0 & 6,7 & 0,0 \\
\hline Augustinópolis & 11.107 & 116 & 10 & 7 & 70,0 & 3 & & & 8,6 & 1,0 & 0,9 \\
\hline Aurora do Tocantins & 4.673 & 435 & & & & & & & 0,0 & 9,3 & 0,0 \\
\hline Axixá do Tocantins & 10.167 & 261 & 13 & 10 & 76,9 & 3 & & & 5,0 & 2,6 & 1,3 \\
\hline Babaçulândia & 9.093 & 336 & 3 & & 0,0 & 3 & & & 0,9 & 3,7 & 0,3 \\
\hline Barrolândia & 6.513 & 400 & & & & & & & & & \\
\hline Bernardo Sayão & 4.369 & 686 & 8 & 2 & 25,0 & 6 & & & 1,2 & 15,7 & 1,8 \\
\hline Brejinho de Nazaré & 6.861 & 600 & 1 & 1 & 100,0 & & & & 0,2 & 8,7 & 0,1 \\
\hline Buriti do Tocantis & 8.280 & 389 & 21 & 11 & 52,4 & 10 & & & 5,4 & 4,7 & 2,5 \\
\hline Caseara & 2.812 & 1.397 & 155 & 71 & 45,8 & 84 & & & 11,1 & 49,7 & 55,1 \\
\hline Colinas do Tocantins & 21.302 & 241 & 58 & 22 & 37,9 & 34 & 2 & & 24,1 & 1,1 & 2,7 \\
\hline Colméia & 8.605 & 77 & 2 & 1 & 50,0 & 1 & & & 2,6 & 0,9 & 0,2 \\
\hline Combinado & 5.014 & 231 & & & & & & & 0,0 & 4,6 & 0,0 \\
\hline Conceição do Tocantins & 6.142 & 1.863 & 173 & 154 & 89,0 & 19 & & & 9,3 & 30,3 & 28,2 \\
\hline Couto de Magalhães & 6.981 & 279 & 40 & 22 & 55,0 & 18 & & & 14,3 & 4,0 & 5,7 \\
\hline Cristalândia & 11.063 & 844 & 21 & 11 & 52,4 & 10 & & & 2,5 & 7,6 & 1,9 \\
\hline Dianópolis & 14.356 & 1.111 & 46 & 28 & 60,9 & 18 & & & 4,1 & 7,7 & 3,2 \\
\hline Divinópolis do TO & 6.454 & 168 & 13 & 4 & 30,8 & 9 & & & 7,7 & 2,6 & 2,0 \\
\hline Dois Irmãos do TO & 7.931 & 45 & & & & & & & 0,0 & 0,6 & 0,0 \\
\hline Dueré & 4.553 & 649 & 9 & & 0,0 & 9 & & & 1,4 & 14,3 & 2,0 \\
\hline Fátima & 4.849 & 224 & 1 & 1 & 100,0 & & & & 0,4 & 4,6 & 0,2 \\
\hline Figueirópolis & 7.266 & 653 & & & & & & & 0,0 & 9,0 & 0,0 \\
\hline Filadélfia & 11.087 & 532 & 5 & 1 & 20,0 & 4 & & & 0,9 & 4,8 & 0,5 \\
\hline Formoso do Araguaia & 17.716 & 1.617 & 36 & 9 & 25,0 & 27 & & & 2,2 & 9,1 & 2,0 \\
\hline Goianorte & 5.191 & 87 & & & & & & & 0,0 & 1,7 & 0,0 \\
\hline Goiatins & 18.490 & 2.894 & 6 & 3 & 50,0 & 3 & & & 0,2 & 15,7 & 0,3 \\
\hline Guarai & 21.127 & 756 & 38 & 17 & 44,7 & 21 & & & 5,0 & 3,6 & 1,8 \\
\hline Gurupi & 59.692 & 1.108 & 66 & 31 & 47,0 & 35 & & & $6,0^{\circ}$ & 1,9 & 1,1 \\
\hline Itacajá & 14.048 & 1.228 & 1 & & 0,0 & 1 & & & 0,1 & 8,7 & 0,1 \\
\hline Itaguatins & 11.162 & 699 & 20 & 6 & 30,0 & 14 & & & $2 ; 9$ & 6,3 & 1,8 \\
\hline Subtotal & 548.817 & 40.555 & 2.317 & 1.104 & 47,6 & 1.206 & 7 & 0 & 5,7 & 7,4 & 4,2 \\
\hline
\end{tabular}


Carta ao Editor. Marques AC. Dados epidemiológicos de malária na Amazônia, por município, referente a 1992. Revista da Sociedade Brasileira de Medicina Tropical 26:43-59, jan-mar, 1993.

Continuação da Tabela 9 - Tocantins: dados epidemiológicos de malária por município, em 1992.

\begin{tabular}{|c|c|c|c|c|c|c|c|c|c|c|c|}
\hline \multirow{2}{*}{ Municípios } & \multirow{2}{*}{$\begin{array}{l}\text { Populaçāo } \\
\text { IBGE } 1992\end{array}$} & \multicolumn{2}{|c|}{ Lâminas } & \multicolumn{5}{|c|}{ Espécies parasitárias } & \multicolumn{3}{|c|}{ Indices } \\
\hline & & exam & posit & falc & \% falc & vivax & $F+V$ & $\mathbf{M}$ & ILP & IAES & IPA \\
\hline Itaporã do Tocantins & 3.596 & 268 & & & & & & & & 7,5 & 0,0 \\
\hline Lizarda & 4.220 & 427 & & & & & & & 0,0 & 10,1 & 0,0 \\
\hline Miracema do Norte & 21.735 & 533 & 25 & 15 & 60,0 & 10 & & & 4,7 & 2,5 & 1,2 \\
\hline Miranorte & 10.862 & 187 & 4 & 4 & 100,0 & & & & 2,1 & 1,7 & 0,4 \\
\hline Marianápolis do TO & 2.368 & 261 & 16 & 14 & 87,5 & 2 & & & 6,1 & 11,0 & 6,8 \\
\hline Monte do Carno & 6.619 & 919 & 1 & 1 & 100,0 & & & & 0,1 & 13,9 & 0,2 \\
\hline Natividade & 10.377 & 2.276 & 209 & 189 & 90,4 & 20 & & & 9,2 & 21,9 & 20,1 \\
\hline Nazaré & 9.321 & 456 & 9 & 1 & 11,1 & 8 & & & 2,0 & 4,9 & 1,0 \\
\hline Nova Olinda & 7.655 & 209 & 9 & 3 & 33,3 & 6 & & & 4,3 & 2,7 & 1,2 \\
\hline Nova Rosalândia & 3.091 & 146 & 1 & & 0,0 & 1 & & & 0,7 & 4,7 & 0,3 \\
\hline Novo Acordo & 3.876 & 441 & & & & & & & 0,0 & 11,4 & 0,0 \\
\hline Palmas & 29.094 & 1.366 & 105 & 42 & 40,0 & 63 & & & 7,7 & 4,7 & 3,6 \\
\hline Palmeirópolis & 12.184 & 300 & 1 & & 0,0 & 1 & & & 0,3 & 2,5 & 0,1 \\
\hline Paraíso do Tocantins & 29.720 & 702 & 77 & 35 & 45,5 & 41 & 1 & . & 11,0 & 2,4 & 2,6 \\
\hline Paraná & 11.092 & 2.209 & 45 & 29 & 64,4 & 16 & & & 2,0 & 19,9 & 4,1 \\
\hline Pedro Afonso & 13.676 & 1.719 & 1 & & 0,0 & 1 & & & 0,1 & 12,6 & 0,1 \\
\hline Peixe & 12.804 & 2.233 & 69 & 17 & 24,6 & 52 & & & 3,1 & 17,4 & 5,4 \\
\hline Pequizeiro & 3.273 & 106 & 2 & 2 & 100,0 & & & & 1,9 & 3,2 & 0,6 \\
\hline Pindorama do Tocantins & 4.940 & 677 & 1 & & 0,0 & 1 & & & 0,1 & 13,7 & 0,2 \\
\hline Pium & 9.033 & 883 & 15 & 12 & 80,0 & 3 & & & 1,7 & 9,8 & 1,7 \\
\hline Ponte Alta do B. Jesus & 6.749 & 553 & & & & & & & 0,0 & 8,2 & 0,0 \\
\hline Ponte Alta do TO & 7.465 & 519 & 2 & 2 & 100,0 & & & & 0,4 & 7,0 & 0,3 \\
\hline Porto Alegre do TO & 2.126 & 144 & 3 & 3 & 100,0 & & & & 2,1 & 6,8 & 1,4 \\
\hline Porto Nacional & 44.621 & 2.695 & 49 & 23 & 46,9 & 25 & 1 & & 1,8 & 6,0 & 1,1 \\
\hline Praia Norte & 5.185 & 413 & 8 & 3 & 37,5 & 5 & & & 1,9 & 8,0 & 1,5 \\
\hline Presidente Kennedy & 6.186 & 165 & & & & & & & 0,0 & 2,7 & 0,0 \\
\hline Rio Sono & 7.089 & 522 & 2 & 1 & 50,0 & 1 & & & 0,4 & 7,4 & 0,3 \\
\hline Samapaio & 4.387 & 221 & 27 & 7 & 25,9 & 20 & & & 12,2 & 5,0 & 6,2 \\
\hline Santa Rosa do TO & 3.896 & 541 & 4 & 3 & 75,0 & 1 & & & 0,7 & 13,9 & 1,0 \\
\hline Santa Tereza do TO & 3.276 & 174 & & & & & & & 0,0 & 5,3 & 0,0 \\
\hline São Sebastião do TO & 4.364 & 303 & 13 & 3 & 23,1 & 10 & & & 4,3 & 6,9 & 3,0 \\
\hline S. Valério da Natividade & 3.748 & 246 & 17 & 6 & 35,3 & H & & & 6,9 & 6,6 & 4,5 \\
\hline Silvanópolis & 6.238 & 225 & & & & & & & 0,0 & 3,6 & 0,0 \\
\hline Sítio Novo do TO & 9.116 & 359 & 8 & 2 & 25,0 & 6 & & & 2,2 & 3,9 & 0,9 \\
\hline Taquatinga & 11.527 & 1.564 & & & & & & & 0,0 & 13,6 & 0,0 \\
\hline Tocantínia & 6.503 & 224 & & & & & & & 0,0 & 3,4 & 0,0 \\
\hline Tocantinópolis & 31.057 & 1.212 & 60 & 11 & 18,3 & 48 & 1 & & 5,0 & 3,9 & 1,9 \\
\hline Wanderlândia & 9.413 & 83 & 3 & 1 & 33,3 & 2 & & & 3,6 & 0,9 & 0,3 \\
\hline Xambioá & 11.773 & 1.666 & 225 & 96 & 42,7 & 129 & & & 13,5 & 14,2 & 19,1 \\
\hline Subtotal & 394.255 & 28.147 & 1.011 & 525 & 51,9 & 483 & 3 & & 3,6 & 7,1 & 2,6 \\
\hline Total & 943.072 & 68.702 & 3.328 & 1.629 & 48,9 & 1.689 & 10 & & 4,8 & 7,3 & 3,5 \\
\hline Ilha do Bananal & $\ldots .$. & 507 & 39 & 24 & 61,5 & 15 & & 0 & 7,7 & $\ldots \ldots$ & $\ldots \ldots$ \\
\hline
\end{tabular}

